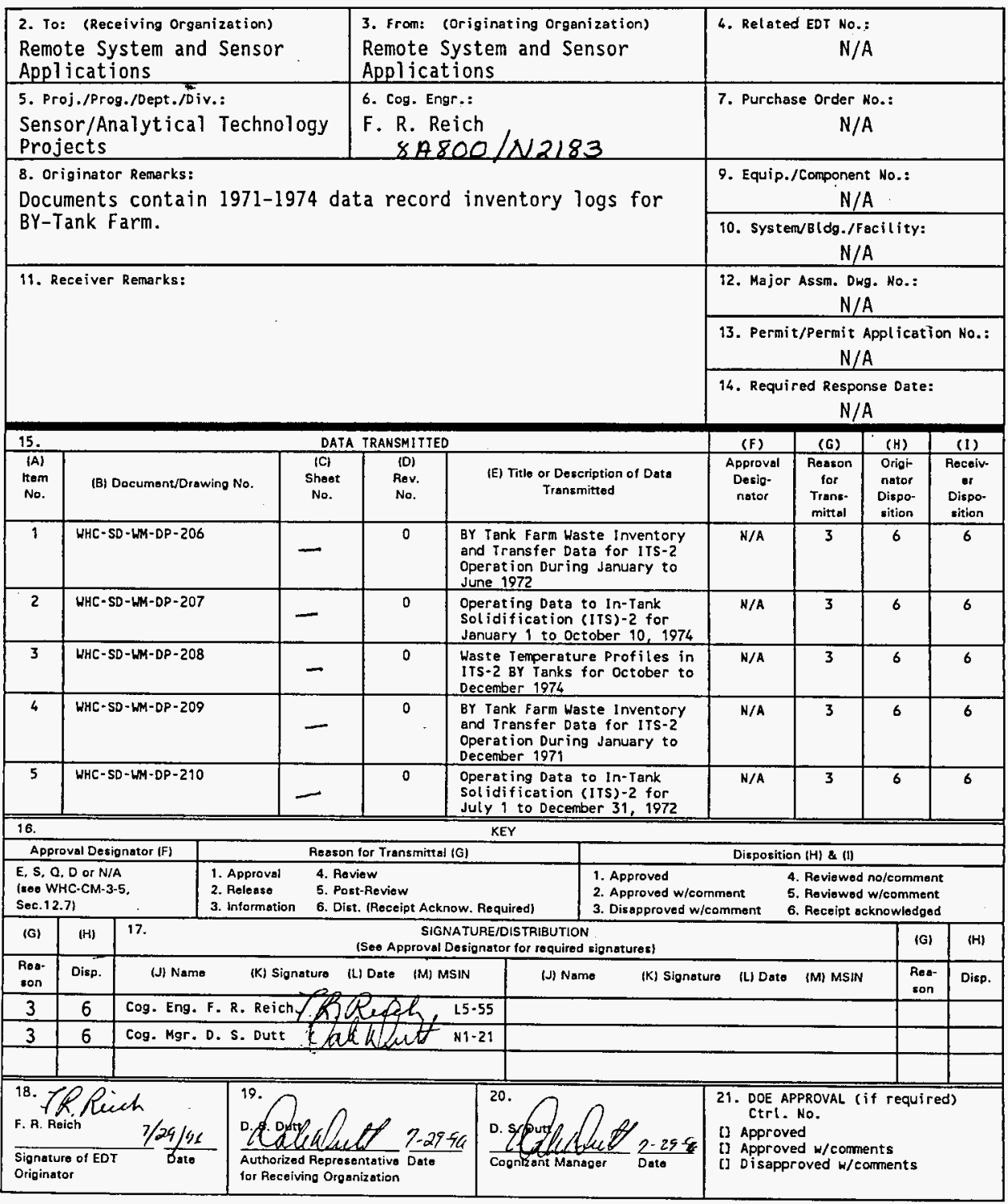




\title{
Waste Temperature Profiles in ITS-2 BY Tanks for October to December 1974
}

\author{
F. R. Reich
}

Westinghouse Hanford Company, Richland, WA 99352

U.S. Department of Energy Contract DE-AC06-87RL10930

\begin{tabular}{|c|c|c|}
\hline $\begin{array}{l}\text { T/ECN: } \\
\text { g Code: } \\
\text { R Code: }\end{array}$ & $\begin{array}{l}617410 \\
8 A 800 \\
\text { EW4010000 }\end{array}$ & $\begin{array}{l}\text { UC: } 721 \\
\text { Charge Code: } \\
\text { Tota1 Pages: }\end{array}$ \\
\hline
\end{tabular}

Key Words: ITS-2, BY tank temperatures/data, temperature, profile Abstract: Data sheets containing profile temperature in ITS-2 bottom. Waste temperature profiles in ITS-2 BY Tanks for October to December 1974.

\section{BEST AVALIBBLE COPY}

TRADEMARK DISCLAIMER. Reference herein to any specific commercial product, process, or service by trade name, tradenark, manufacturer, or otherwise, does not necessarily constitute or imply its endorsement, recomendation, or favoring by the United States Government or any agency thereof or its contractors or subcontractors.

Printed in the United States of America. To obtain copies of this document, contact: WHC/BCs Document Control Services, P.0. Box 1970, Mailstop H6-08, Richland WA 99352, Phone (509) 372-2420; Fax (509) 376-4989.
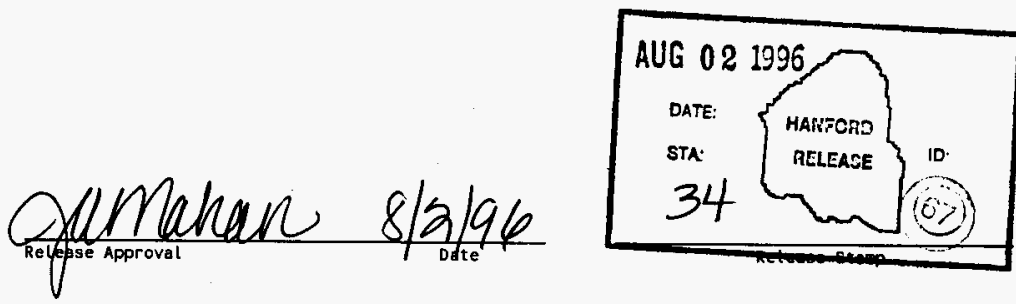

Approved for Public Release 
Document Date: None given

$$
\text { Box 58102 }
$$

Document Number: Nowe given

RHA Box Number: 5810

File Number: None giluen

Title:

PROFILE TEMPERATURE IN ITS-2 BOTTOM

Description:

49 PAGES PAPER CLIPPED. PROFILE TEMPERATURE IN ITS-2 BOTTOM 10-74 TO 12-74. STORED WITHIN A FILE FOLDER

\section{Comments:}

TEMPERATURES TAKEN AT DIFFERENT DEPTHS OF TANK

Addressee, Company:

Author:

Tank \# :

241-BY FARM

Keywords:

PROFILE TEMPERATURES

References: $N$

\# References: None given

Action Required: N

Topic: None given

Action Taken: None given

User: RM SCHWARZ

User Datē: 02-04-92 07:50:44

Reviewer: ECKSTEIN D

Review Date: 09-16-91

Checked By: SCHWARZ RM

Checked Date: 10-10-91

WTS Key: 964

Document Type: DS
Company:

$$
\begin{aligned}
& \text { Thun } \\
& \text { Exilam } \\
& \text { buth }
\end{aligned}
$$

$$
\begin{aligned}
& 102 \\
& 103-10124 / 24 \\
& 104- \\
& 105- \\
& 106- \\
& 10 ? \\
& 108 \\
& 104- \\
& 110 \\
& 111- \\
& 112
\end{aligned}
$$




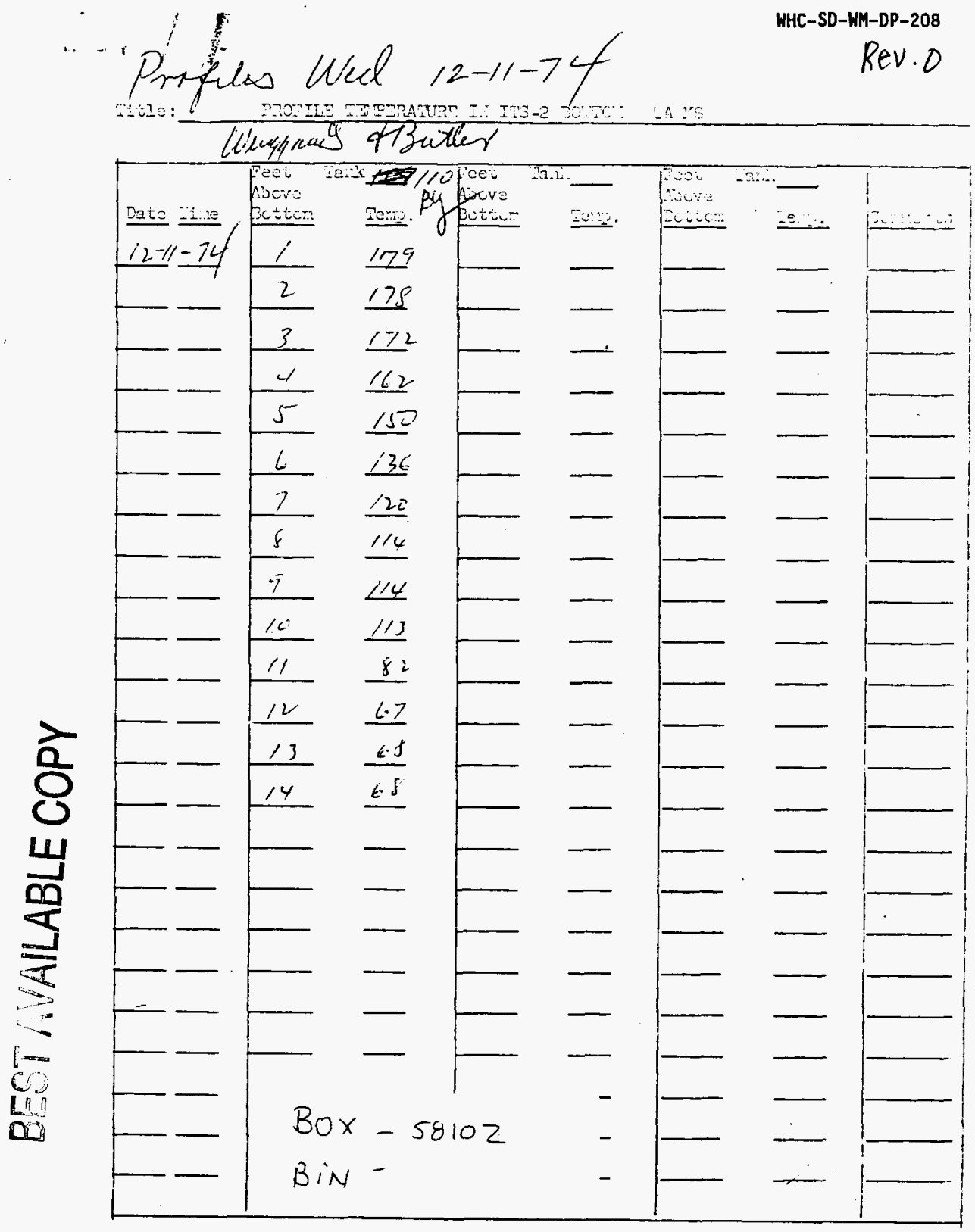

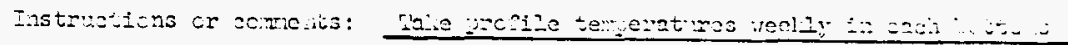

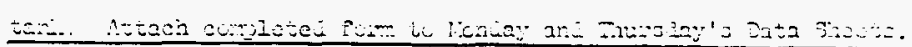


misle:

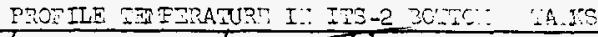
Mlequands of Buller

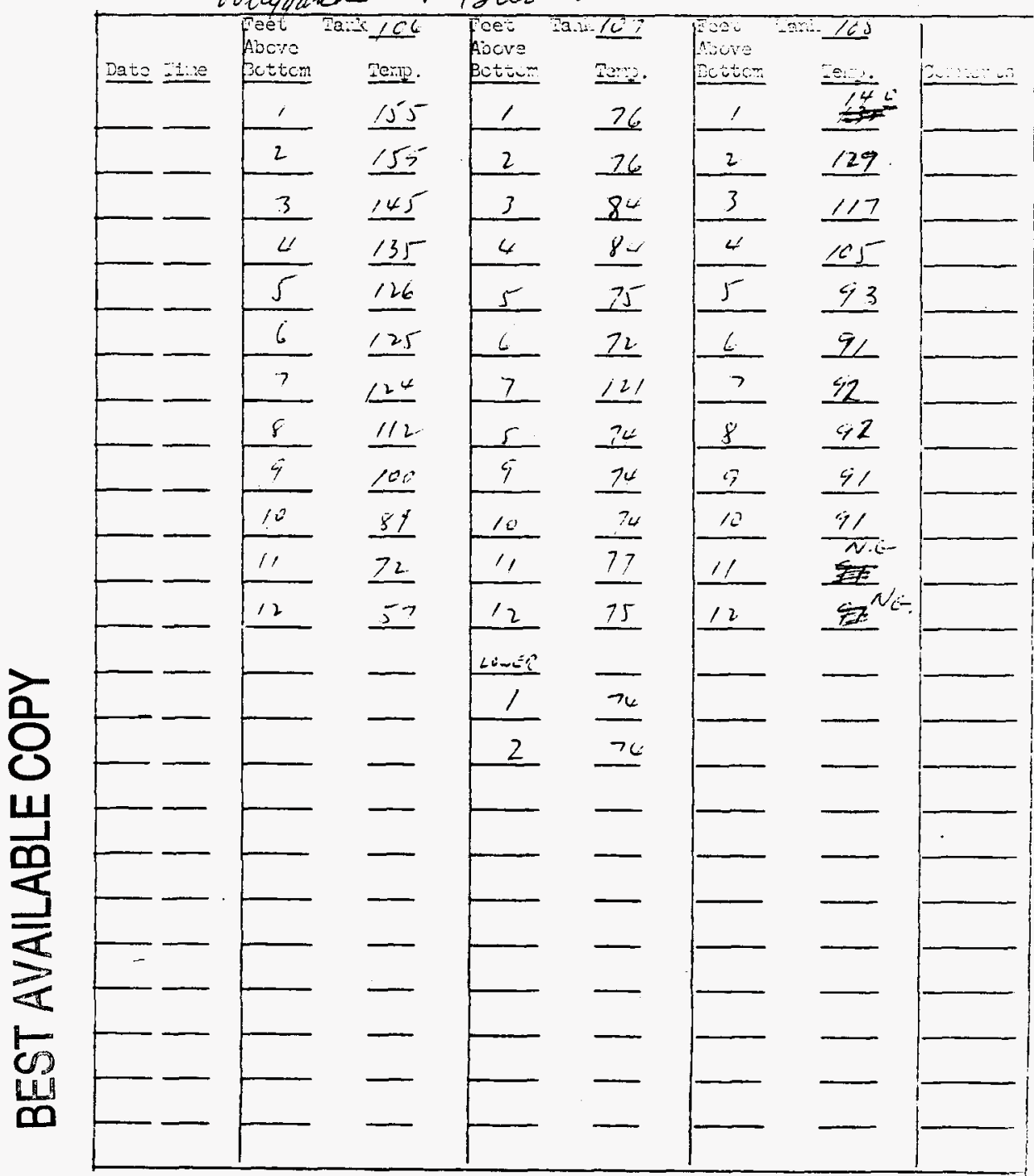

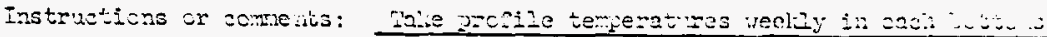




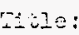

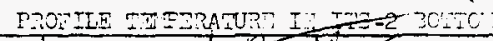

A. Jrs

Whequand of Buthr

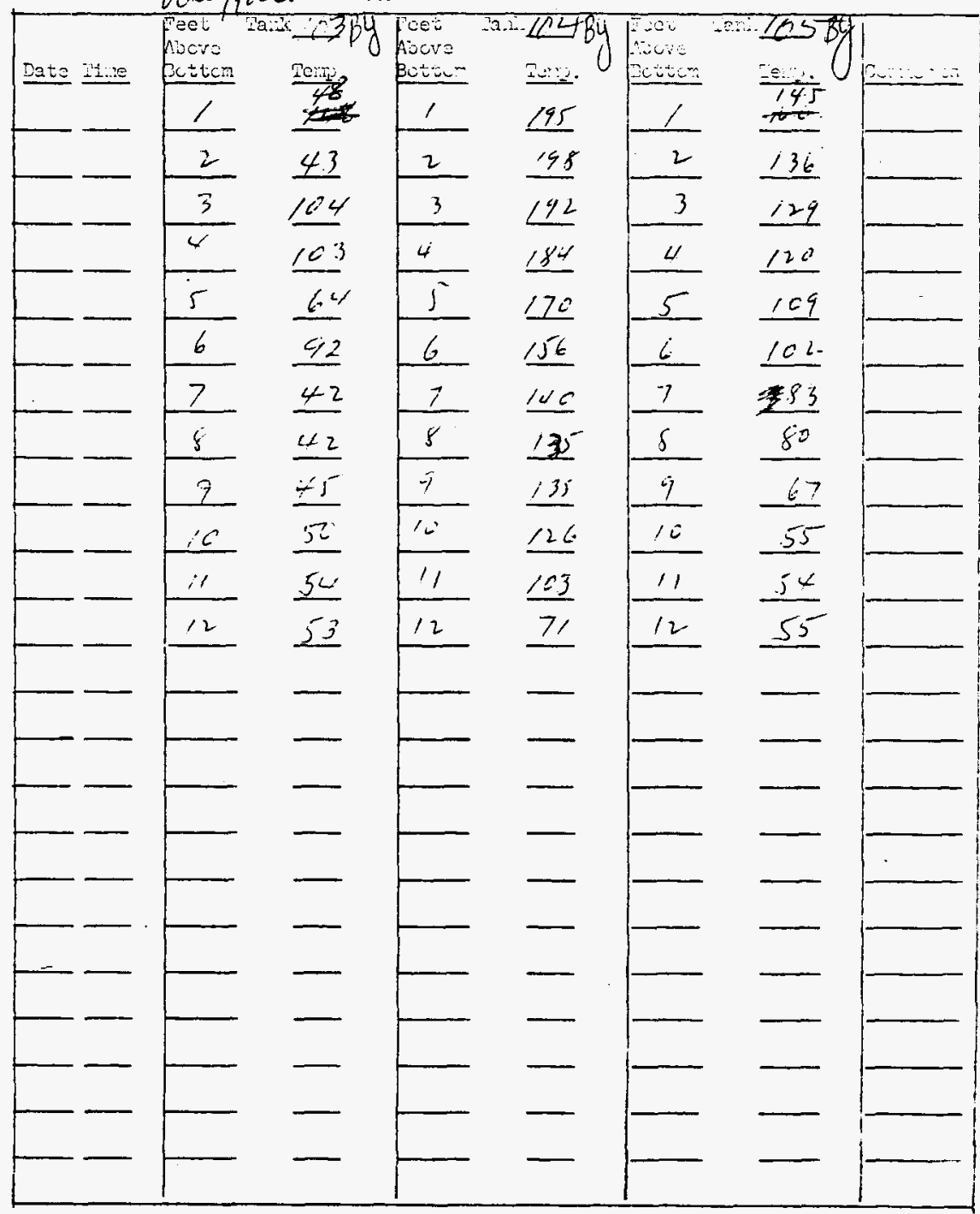

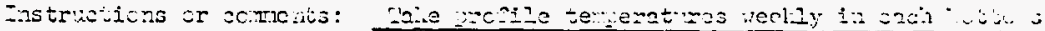

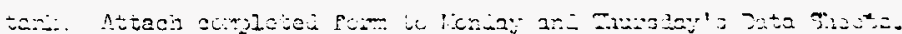


iol, $\quad \therefore+1=$

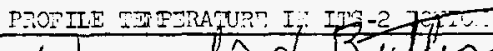

107

108

109

110

, 12
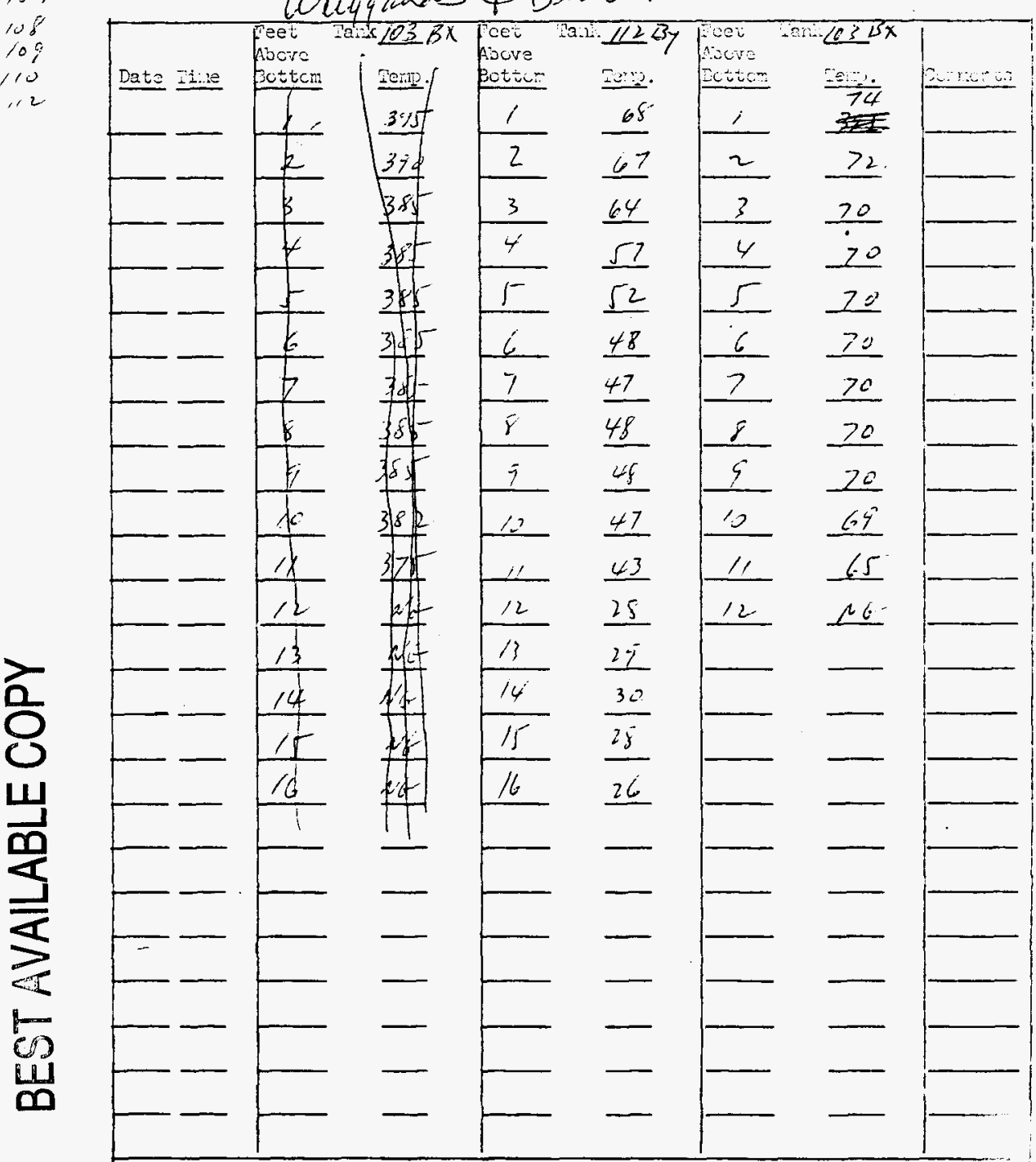

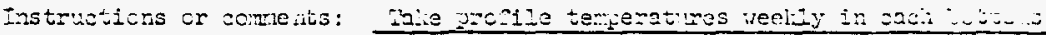




\section{$2+610:$}

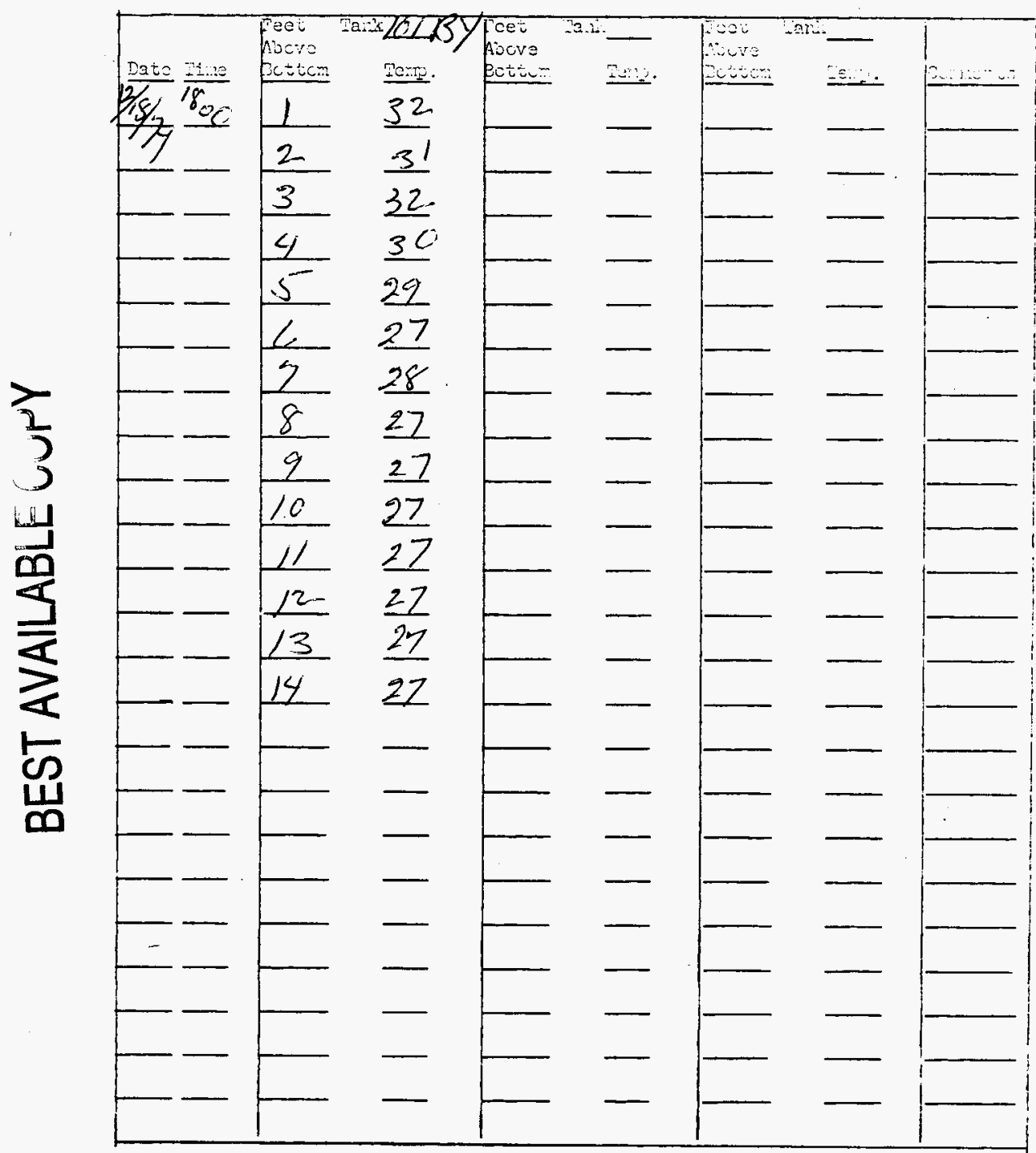

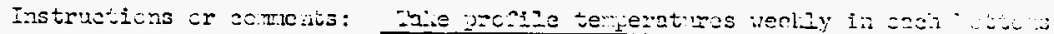


Tile:

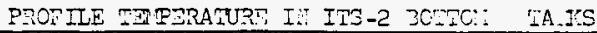

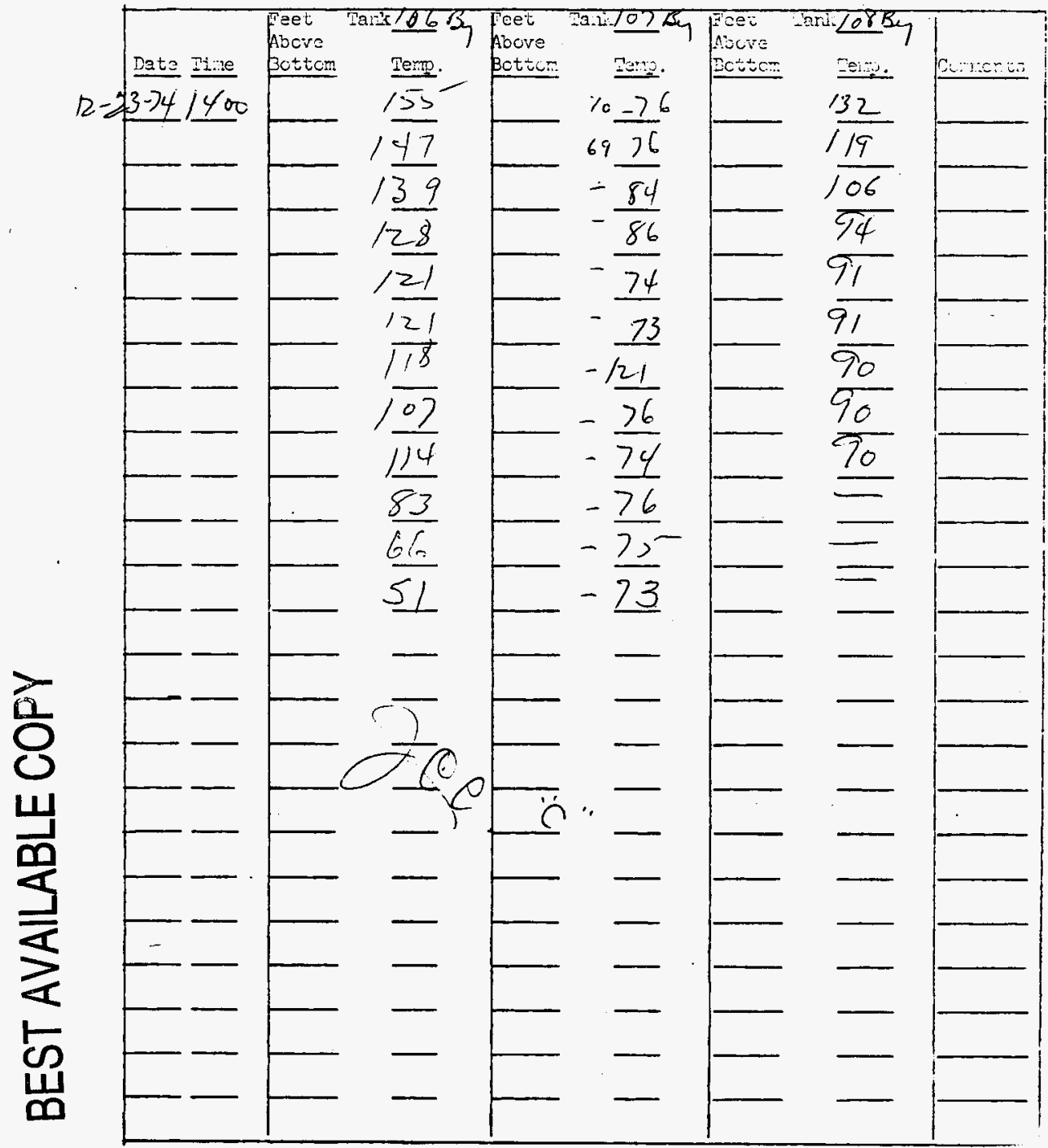

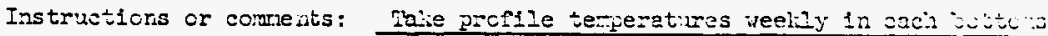
tani. Attach completec fom to lioniay ari Tursday'z Data Sieatz. 
r+iำ:

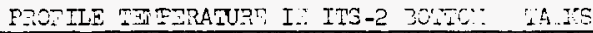

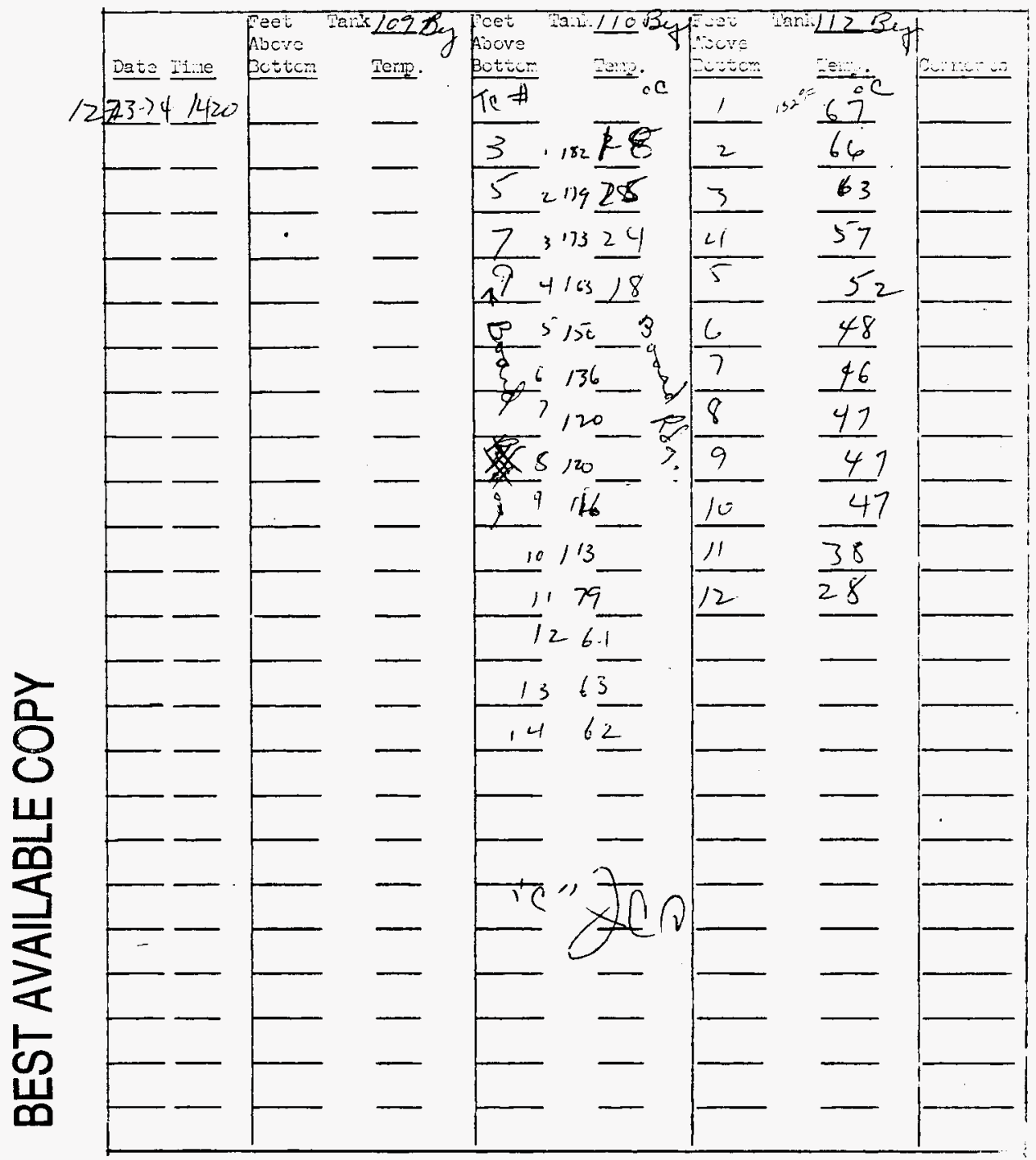

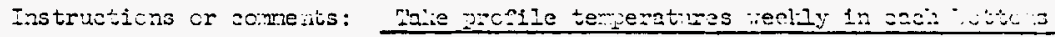

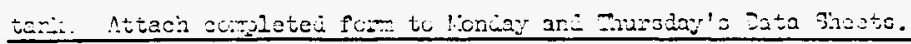


Fis :

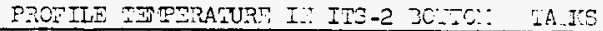

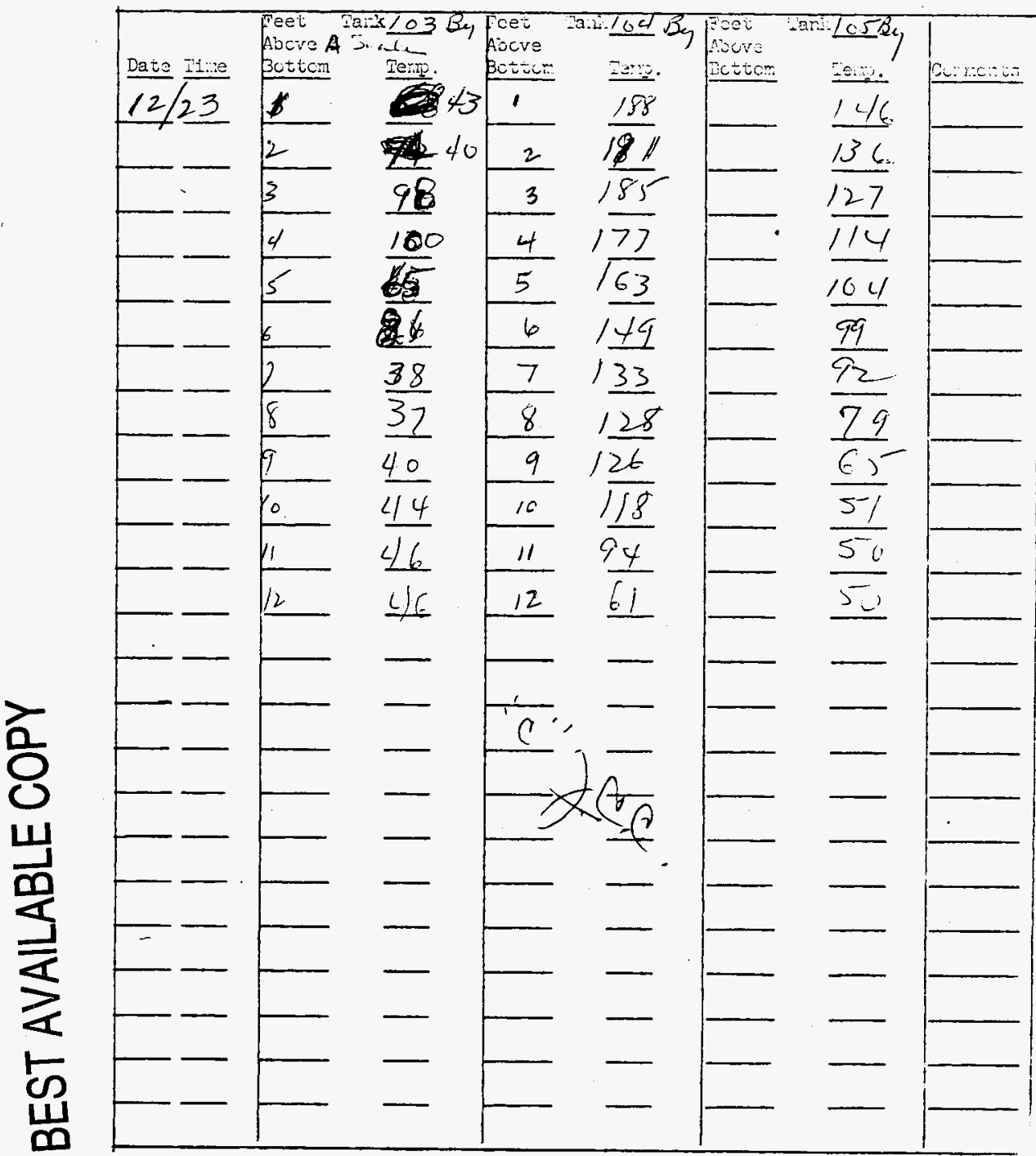

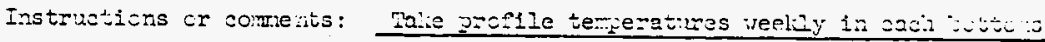

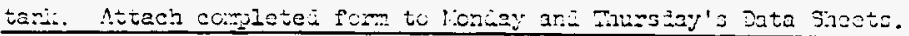


İ⿴囗十:

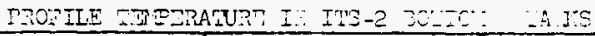

$B Y-$

peel pack l6 $3 B$ p

Fians

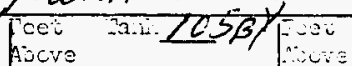

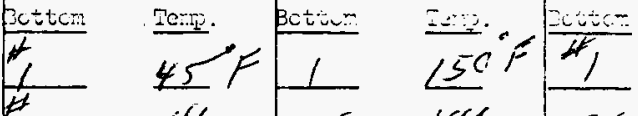

$12+7.74=$

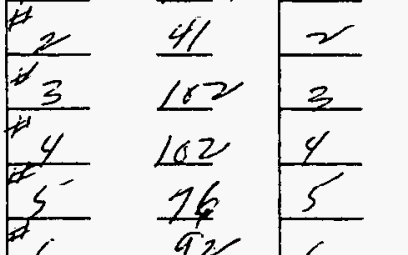

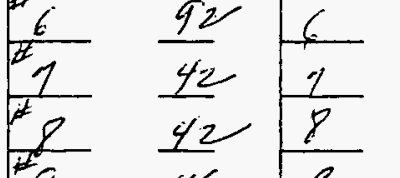

然

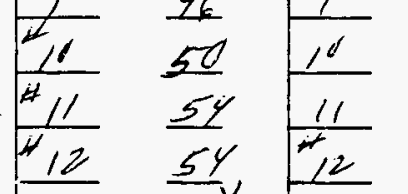

$12-17-7 y$

a

ᄋ

U

$\overline{\mathbf{m}}$

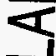

$\sum$

co

岗

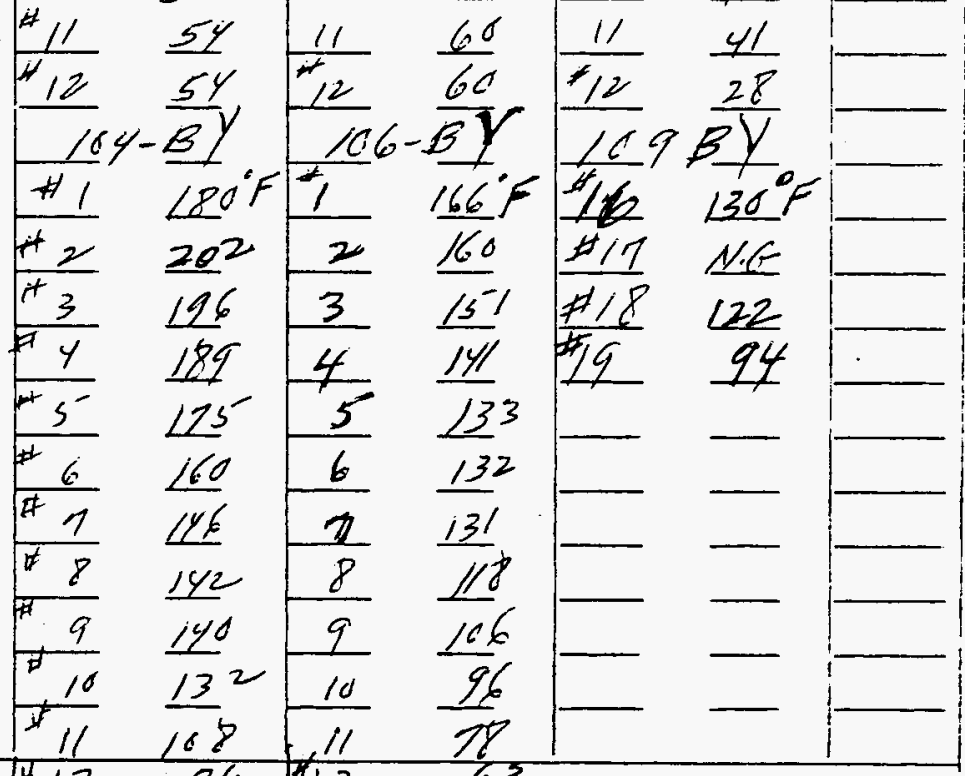

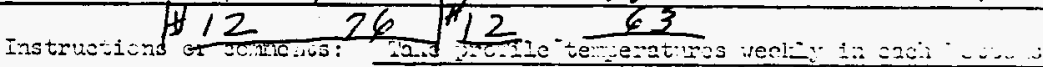

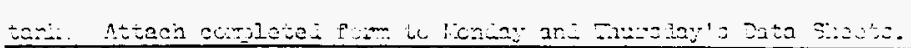


$2010:$

PAOTILE MOERAIU?? IN ITS-2 $3 \mathrm{C}+\mathrm{C}$

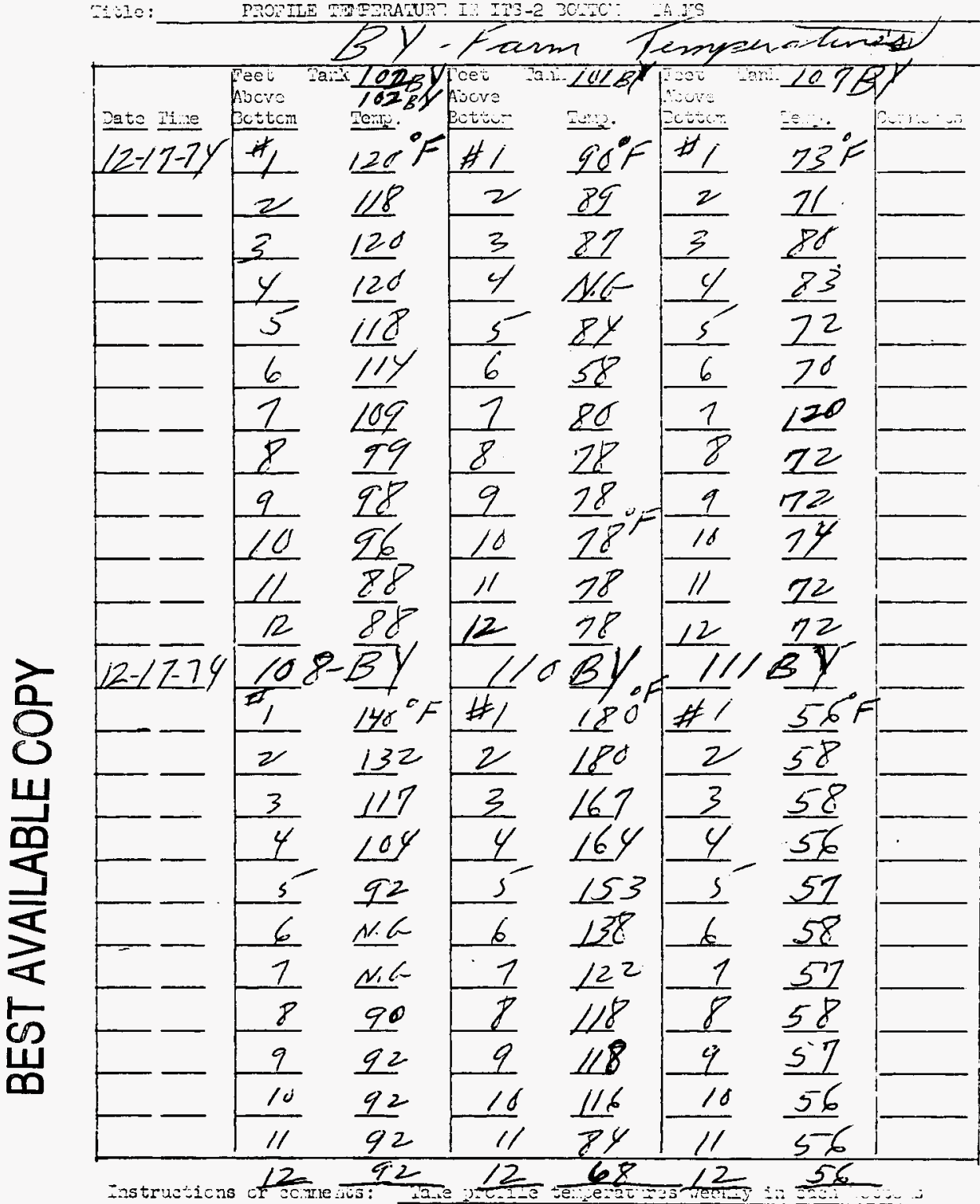

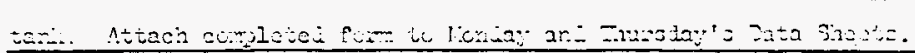


tons:

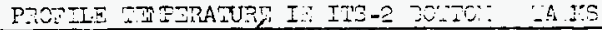

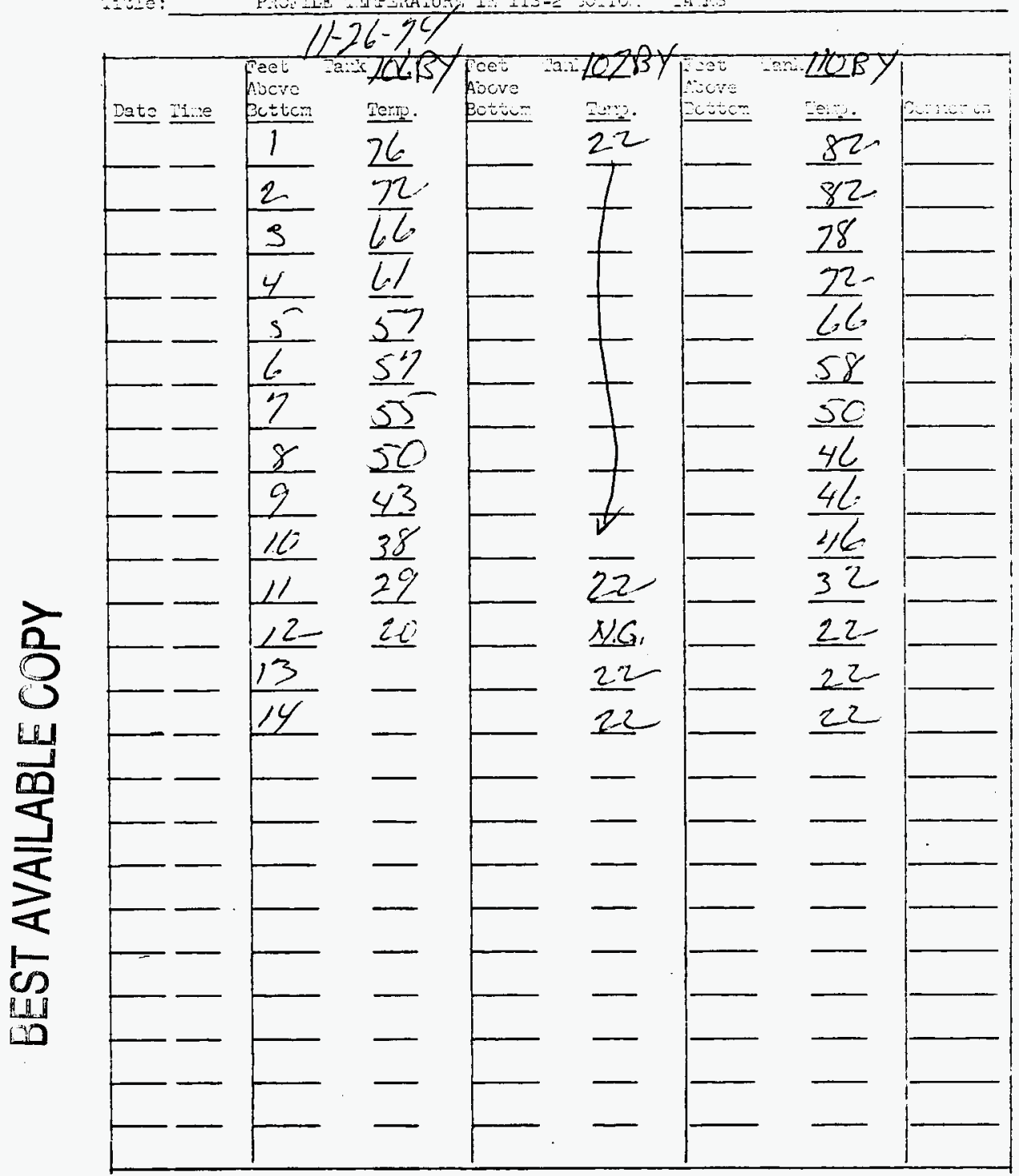

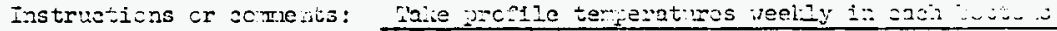

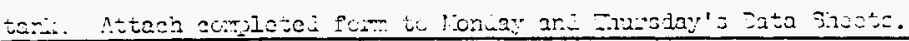




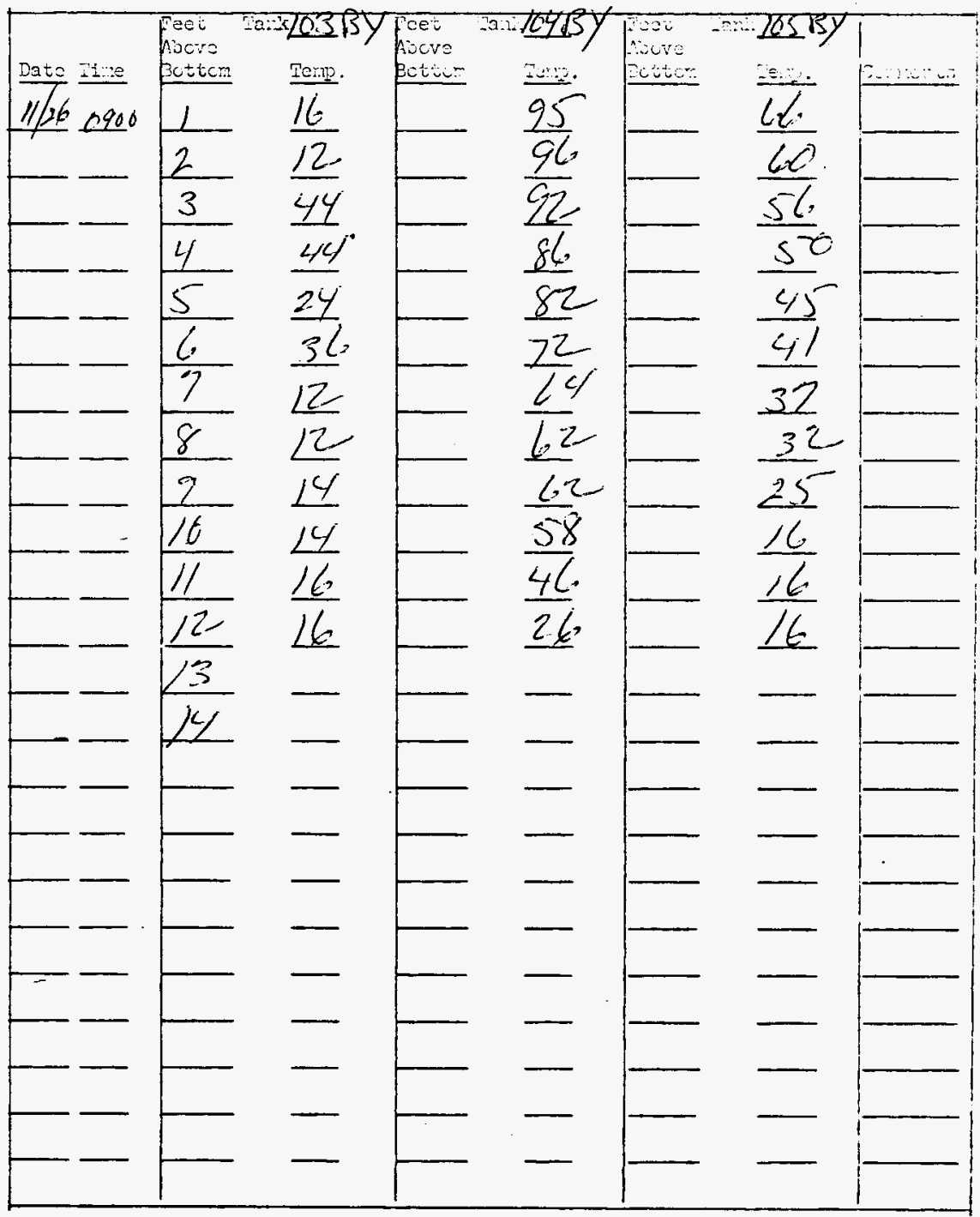

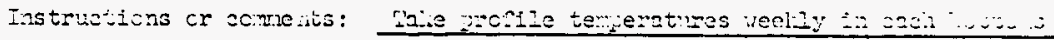

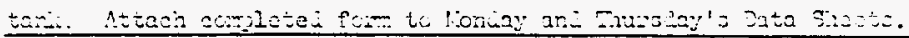


7010: PROTIIE TEZBATURT IA IIS-2 30,

4.5
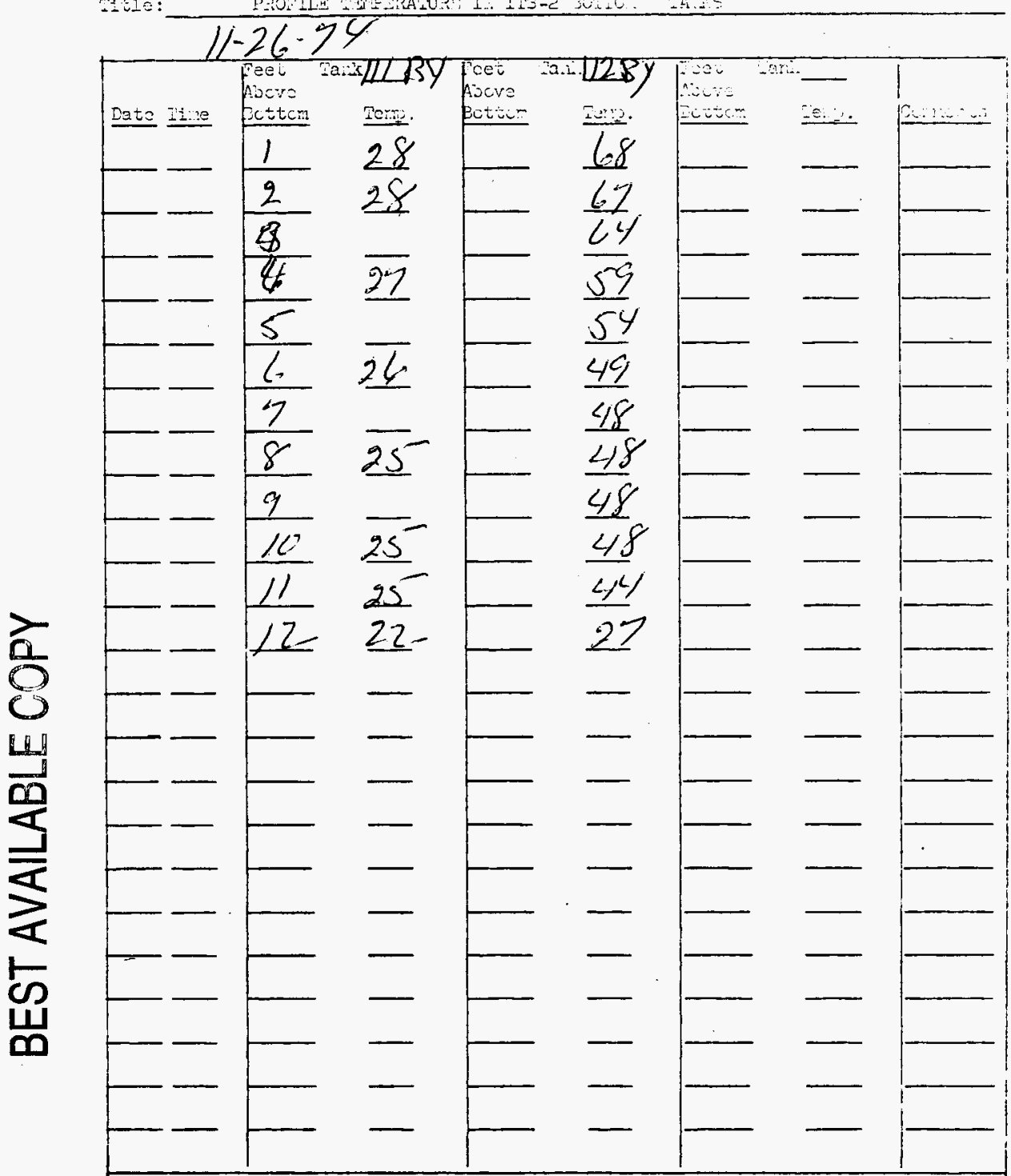

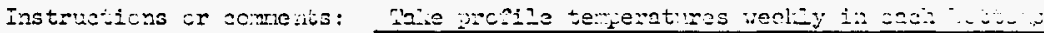

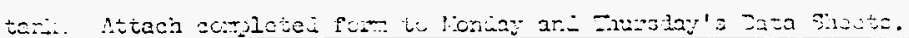


M:Lic:

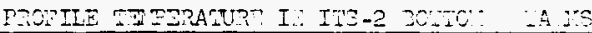

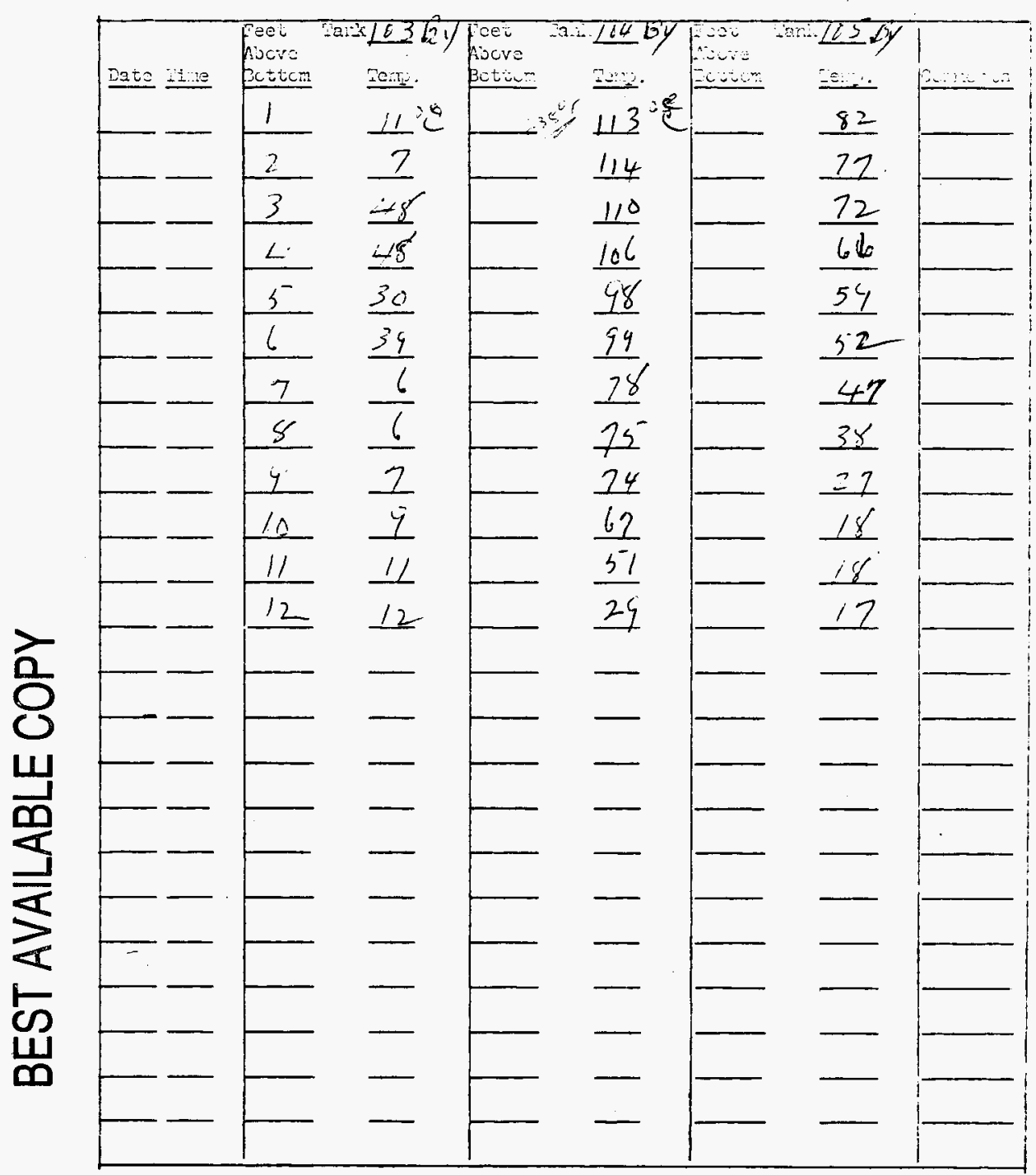

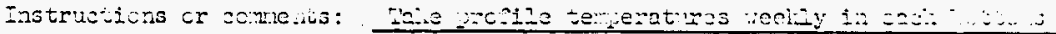

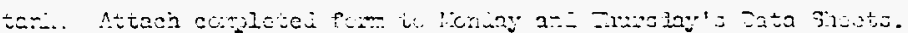



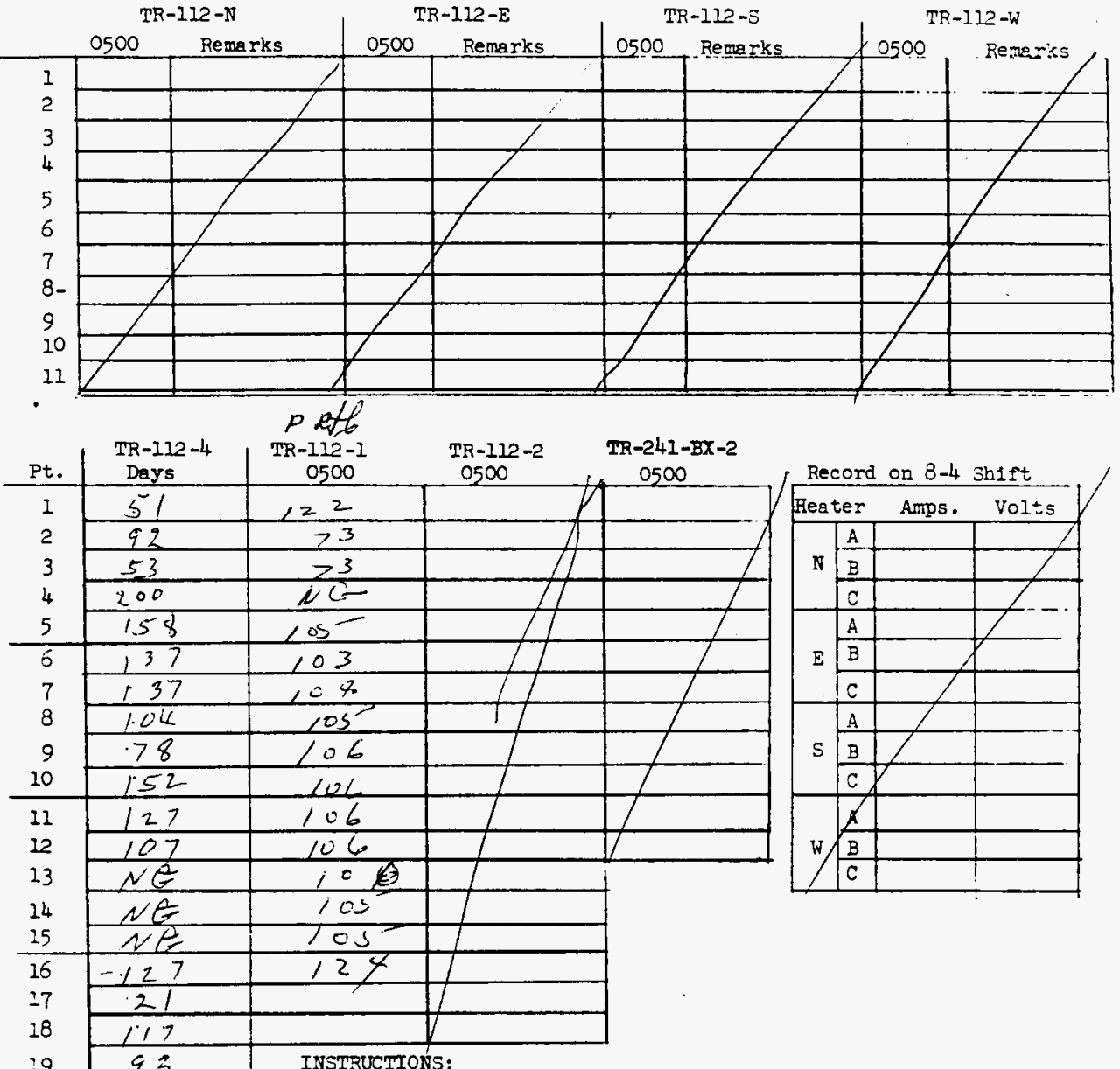

1. FIII in all blanks

2. If data are not avallable, give reason under Remarks.

3. If recording instruments, the rmocouples, etc. are not runctioning properly, report this to supervision.

4. Circle all results that are out of process limits or are otherwise questionable.

5. All suggestions, formal or 1nformal, w1ll be given prompt attention. 
ITS-2 TEMPERATURES
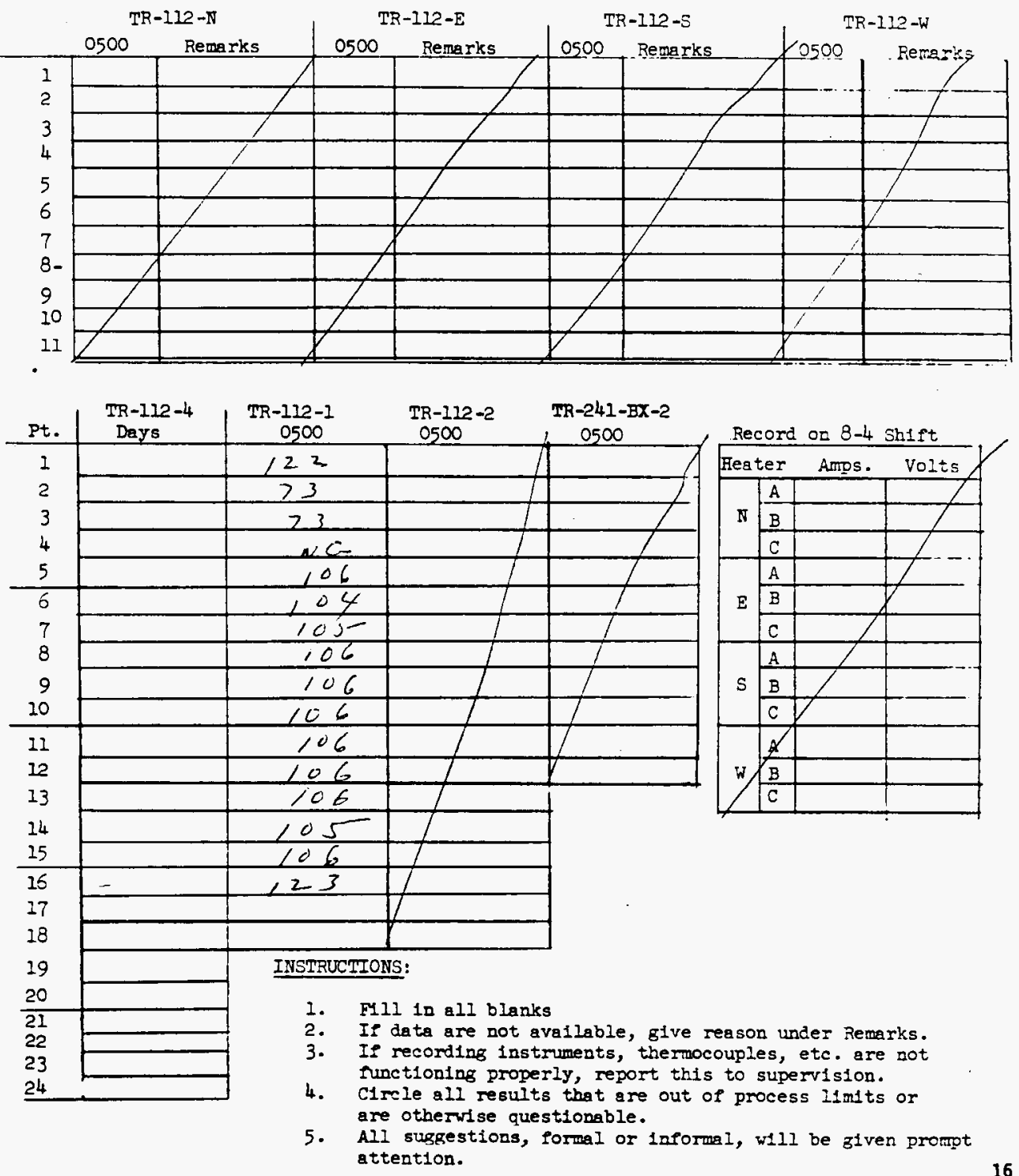

Rev1sed 3-23-73 

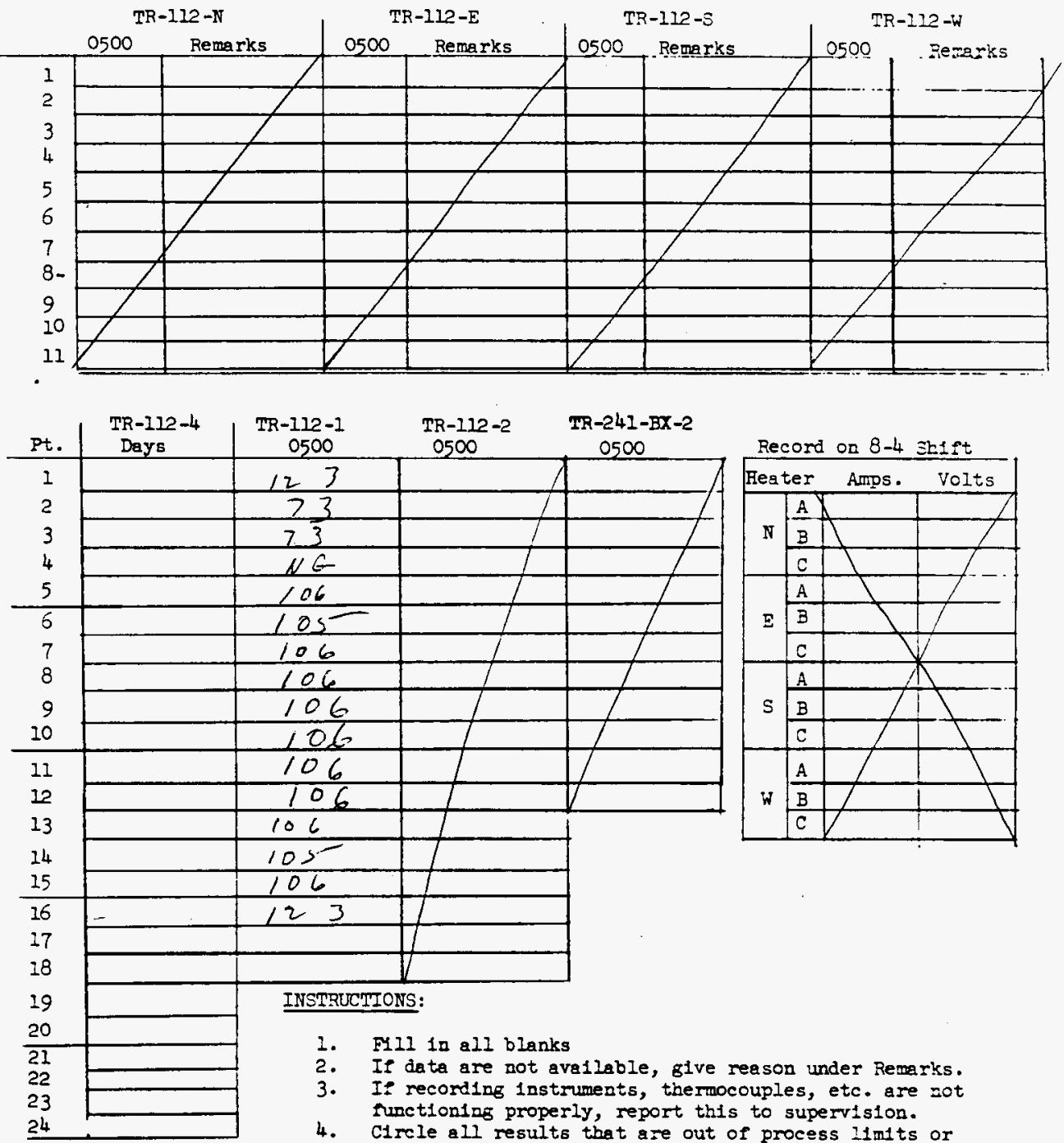

TR-112-1 TR-112-2 TR-24I-BX-2 $0500 \quad 0500 \quad 0500$ \begin{tabular}{c|c|c|}
123 & 0 \\
\hline 73 & & \\
73 & &
\end{tabular} NG 106 $105=$ 106 106 106 106 106 106 106 105 106 123

INSTRUCTIONS:

1. Fill in all blanks

2. If date are not avallable, give reason under Remarks.

3. If recording 1nstruments, thermocouples, etc. are not runctionias properly, report this to supervision.

4. Circle all results that are out of process limits or are otherwise questioneble.

5. All suggestions, formal or 1nformal, will be given prompt attention.
Record on 8-4 3h1ft

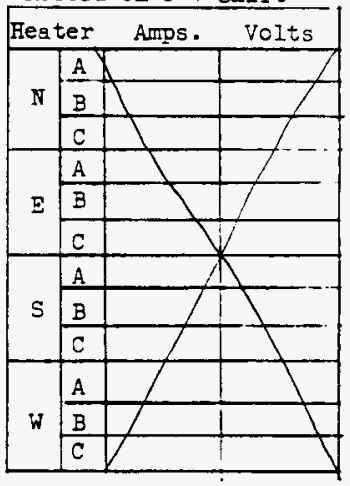



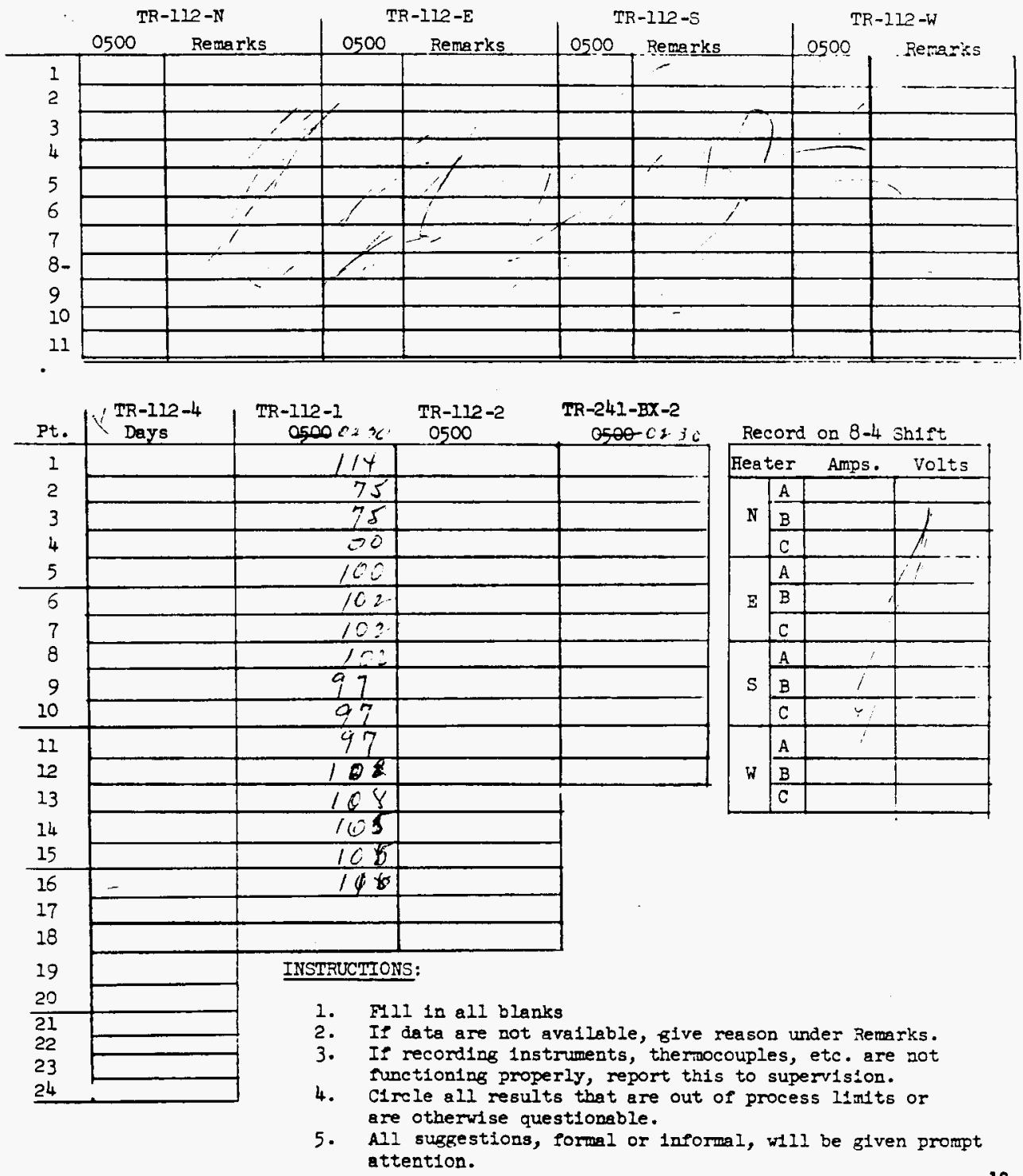


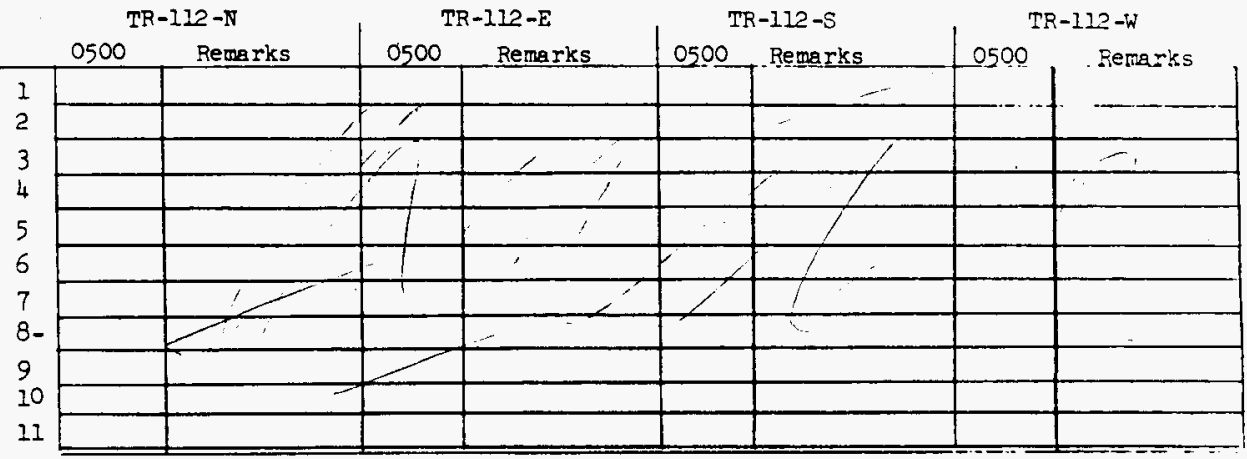

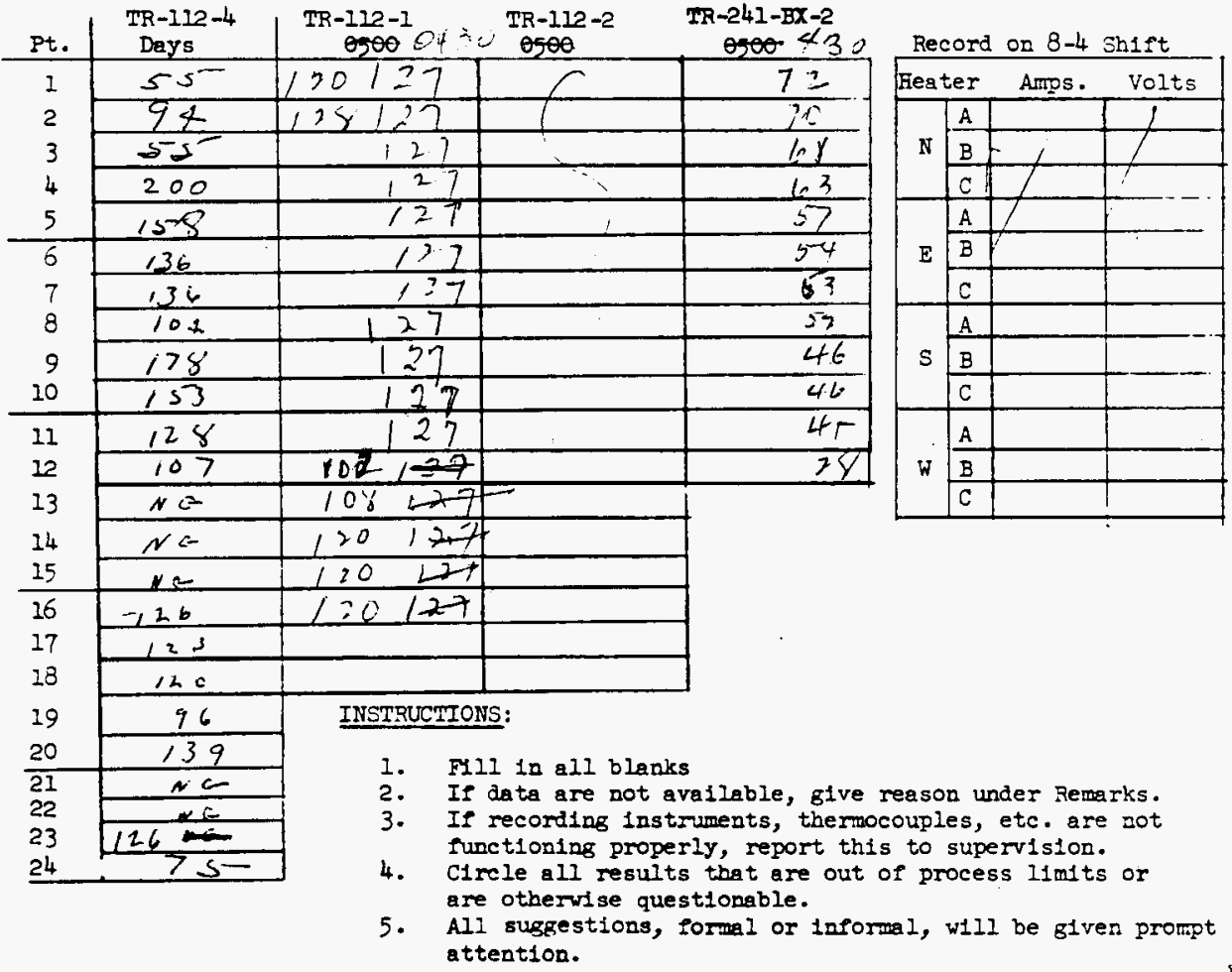



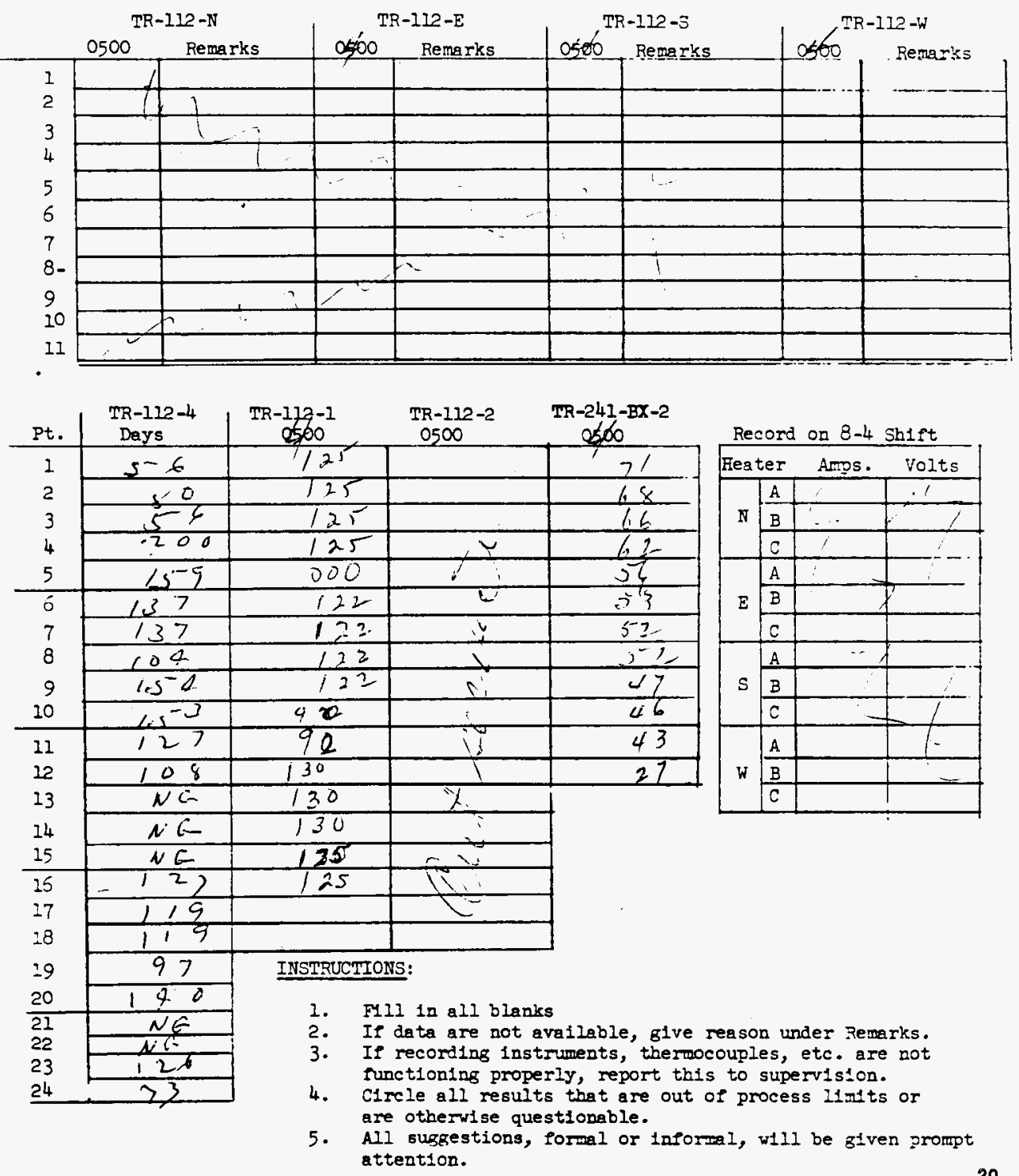
ITS -2 TEMPERATURES

Date:

$11-5-7 \operatorname{Rev}^{\circ}$

$T R-112-\mathrm{N}$

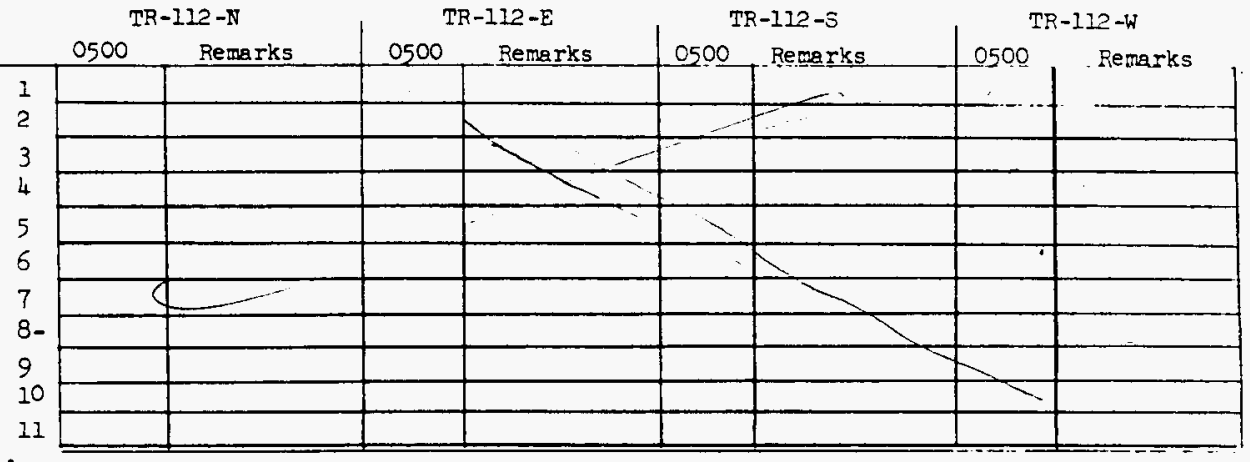

\begin{tabular}{l|c|cc} 
TR-112-4 & TR-112-1 & TR-112-2 & TR-24I-BX-2 \\
Days & 0500 & 0500 & 0500
\end{tabular}

\begin{tabular}{|c|c|c|c|c|c|c|c|c|}
\hline Pt. & Days & 0900 & 0500 & 0900 & \multicolumn{4}{|c|}{ Record on 8-4 Shift } \\
\hline 1 & $5-6$ & 110 & $I$ & 71 & \multicolumn{2}{|c|}{ Feater } & Amps. & Volts \\
\hline 2 & 80 & +26126 & & $\leqslant y$ & \multirow{3}{*}{$\mathrm{N}$} & $\mathrm{A}$ & & \\
\hline 3 & 56 & 125 & I & 66 & & $B$ & & \\
\hline 4 & 200 & 72 & 1 & 62 & & $C$ & & \\
\hline 5 & 159 & 103 & 7 & $j u$ & \multirow{3}{*}{$E$} & A. & & \\
\hline 6 & 137 & 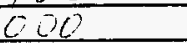 & & 55 & & $B$ & & \\
\hline 7 & 7.37 & 106 & 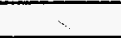 & 53 & & $\mathrm{C}$ & & \\
\hline 8 & 108 & 106 & & 52 & \multirow{3}{*}{$\mathrm{s}$} & A & & \\
\hline 9 & 152 & 106 & & 45 & & $B$ & & \\
\hline 10 & $\left.15^{-}\right)$ & 106 & & $4 k$ & & C & & \\
\hline 11 & 127 & 97 & & 43 & \multirow{3}{*}{ W } & $A$ & & \\
\hline 12 & $10 x$ & 946 & & 27 & & $\bar{B}$ & & \\
\hline 13 & $=$ & 127 & & 1 & & C & & \\
\hline 14 & - & 112 & & & & & & \\
\hline
\end{tabular}

\begin{tabular}{l|l|l|l|}
\hline 15 & & 112 & \\
\hline 16 & -127 & 111 & \\
17 & $1 / 9$ & & \\
\cline { 2 - 4 } 18 & $1 / 9$ & & \\
\cline { 2 - 4 } 19 & 57 & \multicolumn{2}{|c|}{ INSTRUCTIONS: } \\
\cline { 2 - 4 } & &
\end{tabular}

1. FM11 in all blanks

21

140

22

23

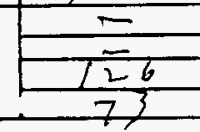

2. If data are not available, give reason under Remarks.

3. If recording instmuments, thernocouples, etc. are not functioning properly, report this to supervision.

4. Circle all results that are out of process lintts or are otherwise questionable.

5. All suggestions, formal or informal, will be given prompt attention. 
ITS -2 TEMPERATURES

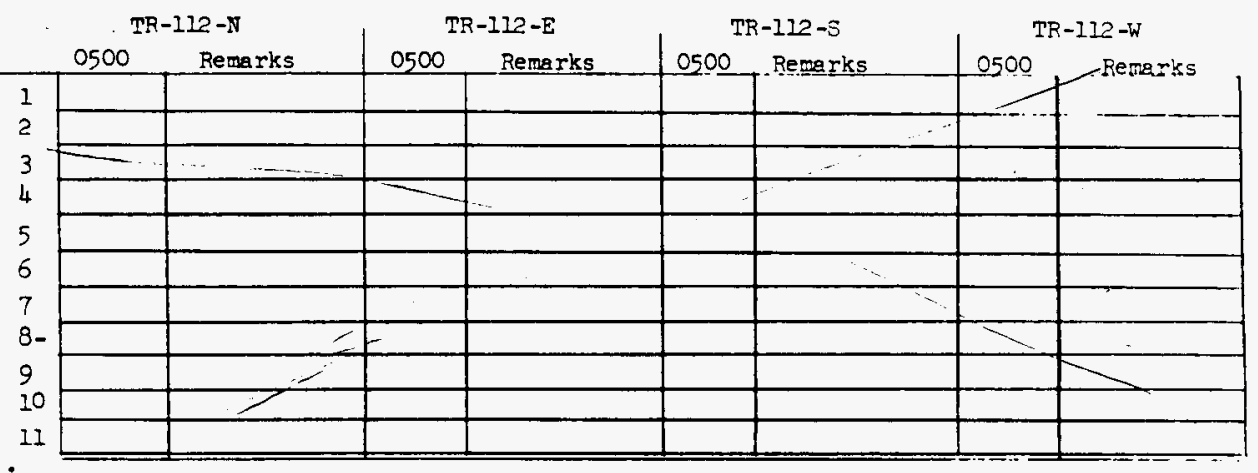

\begin{tabular}{|c|c|}
\hline Pt. & $\begin{array}{l}\text { TR-112- } 4 \\
\text { Days }\end{array}$ \\
\hline 2 & $5-8$ \\
\hline 2 & 75 \\
\hline 3 & $5=8$ \\
\hline 4 & 2 (ब) 0 \\
\hline 5 & 16.0 \\
\hline 6 & 138 \\
\hline 7 & 138 \\
\hline 8 & 105 \\
\hline 9 & 80 \\
\hline 10 & 7.55 \\
\hline 11 & 127 \\
\hline 12 & $\operatorname{lox}$ \\
\hline 13 & 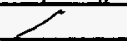 \\
\hline 14 & \\
\hline 15 & 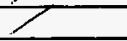 \\
\hline 16 & $-12 \gamma$ \\
\hline 27 & $Y^{\prime 2}$ \\
\hline 18 & 118 \\
\hline 19 & 58 \\
\hline 20 & 140 \\
\hline$\overline{21}$ & \\
\hline $\begin{array}{l}<2 \\
23\end{array}$ & 125 \\
\hline 24 & 70 \\
\hline
\end{tabular}

TR-241-BX-2

TR-112-1 $y$ \% TR-112-2

$0,000+3 ;$

Record on 8-4 Shift

\begin{tabular}{|c|c|c|c|}
\hline \multicolumn{2}{|c|}{ Heater } & Amps. & Volts \\
\hline \multirow{3}{*}{ N } & A & & \\
\hline & $\bar{B}$ & & \\
\hline & $C$ & & \\
\hline \multirow{3}{*}{$E$} & A & & \\
\hline & $B$ & & \\
\hline & C & 7 & \\
\hline \multirow{3}{*}{$S$} & A & 7 & \\
\hline & B & $L$ & \\
\hline & C & & \\
\hline \multirow{3}{*}{ W } & A & & \\
\hline & $B$ & & \\
\hline & C & & \\
\hline
\end{tabular}

INSTRUCTIONS:

1. FIIl in all blanks

2. If data are not avallable, give reason under Remarks.

3. If recording 1nstruments, thermocouples, etc. are not

runctioning properly, report this to supervision.

4. Circle all results thet are out of process 11 mits or are otherwise questionable.

5. All suggestions, formal or 10formal, will be given prompt attention. 

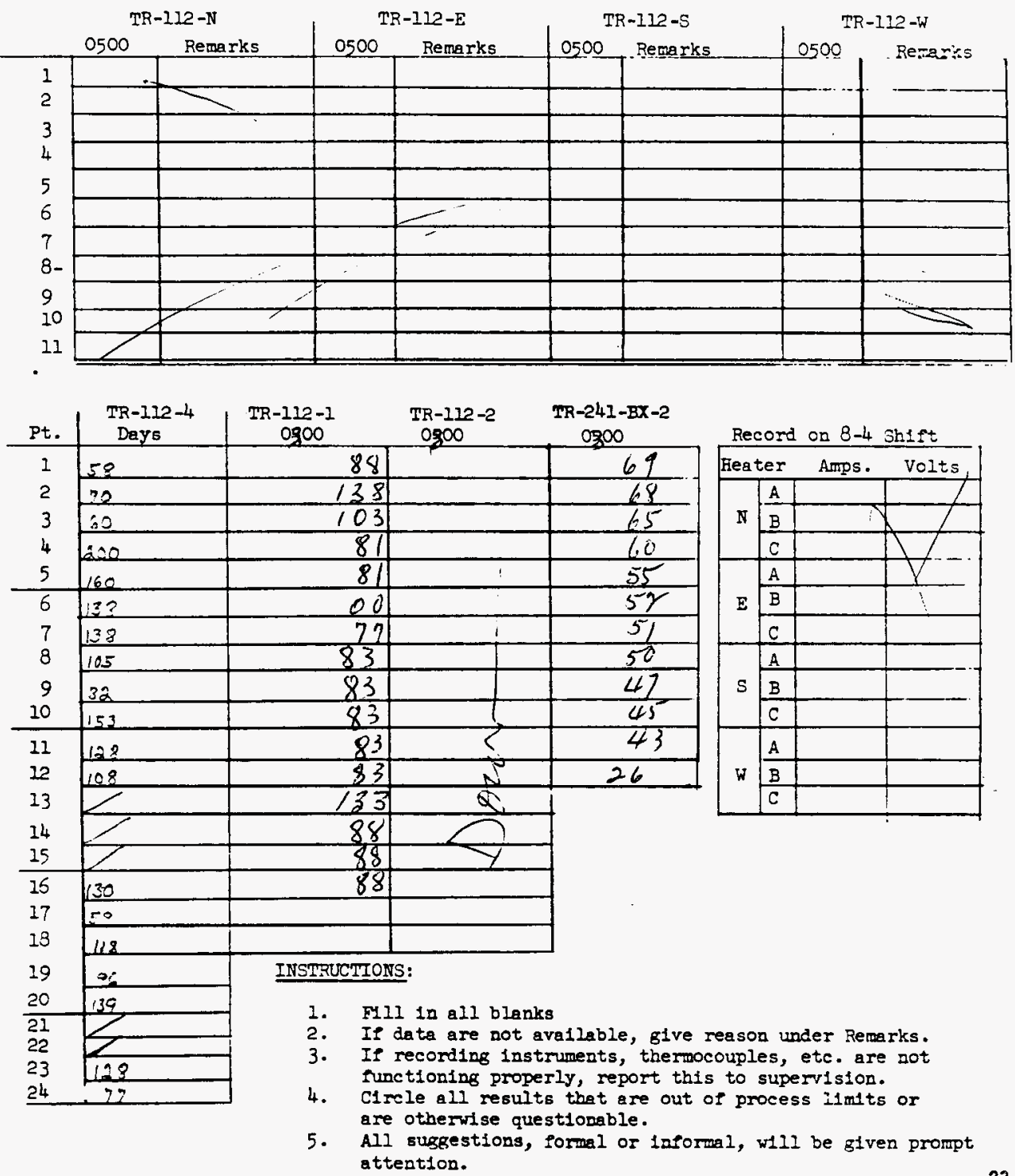


\section{ITS-2 TEMMERATURES}

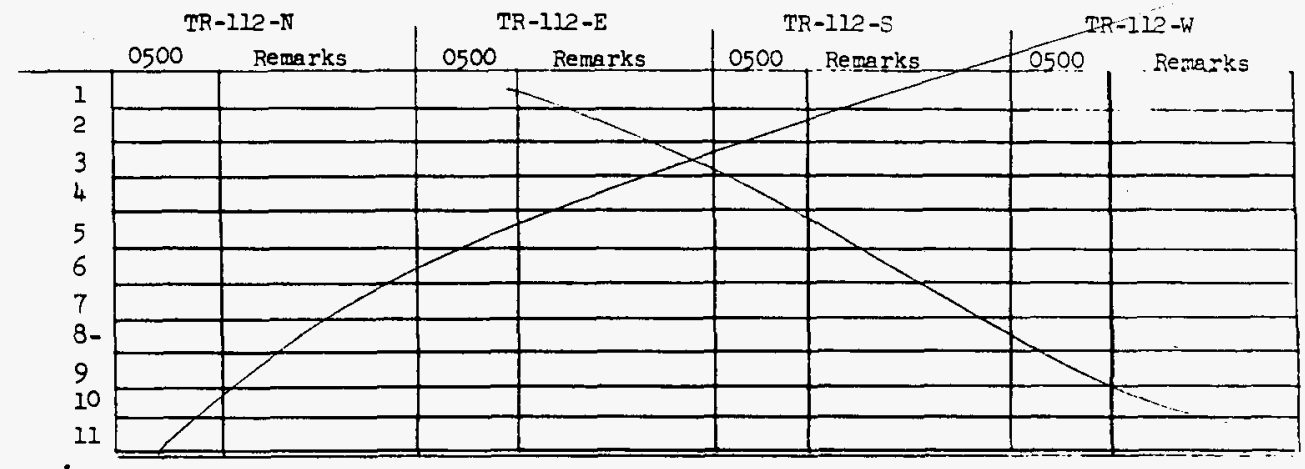

\begin{tabular}{|c|c|c|c|c|}
\hline Pt. & $\begin{array}{l}\text { TR-112-4 } \\
\text { Days }\end{array}$ & $\begin{array}{c}T R-112-1 \\
0300\end{array}$ & $\begin{array}{l}\text { TR-112-2 } \\
0900\end{array}$ & $\begin{array}{l}\mathrm{TR}-241-\mathrm{BX}-2 \\
0300 \\
\end{array}$ \\
\hline 1 & 53 & 95 & & 70 \\
\hline 2 & 69 & 137 & & 64 \\
\hline 3 & 60 & 103 & 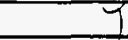 & 66 \\
\hline 4 & 200 & 80 & 302 & 61 \\
\hline 5 & 159 & 40 & 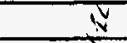 & $\$ 5$ \\
\hline 6 & 138 & 0 & 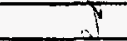 & $5 ?$ \\
\hline 7 & 173 & 92 & (i) & 52 \\
\hline 8 & 104 & 92 & I & 52 \\
\hline 9 & 82 & 92 & $x$ & 45 \\
\hline 10 & $:=4$ & 92 & 7 & 46 \\
\hline 11 & 128 & 92 & $n+$ & 43 \\
\hline 12 & 108 & 92 & 51 & 27 \\
\hline 13 & $\angle$ & 130 & N & \\
\hline 14 & 2 & io & 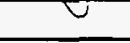 & \\
\hline 15 & 2 & 95 & & \\
\hline 15 & 119 & 95 & & \\
\hline 17 & $\therefore 8$ & & & \\
\hline 18 & L19 & & & \\
\hline 19 & 96 & \multicolumn{2}{|c|}{ INSTRUCTIONS: } & \\
\hline
\end{tabular}

Record on 8-4 Sh1ft

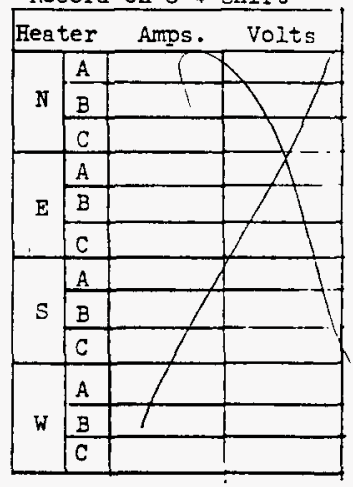

1. F111 in all blanks

2. If data are not avallable, give reason under Remarks.

3. If recording instruments, themocouples, etc. are not runctioning properly, report this to supervision.

4. Circle all results that are out of process limits or are otherwlse questionable.

5. All suggestions, formel or informal, will be given prompt attention. 
ITS-2 TMMPERATURES

WHC-SD-WM-DP-208

Dete :

$1 / 1-7 \mathrm{ReNO}$

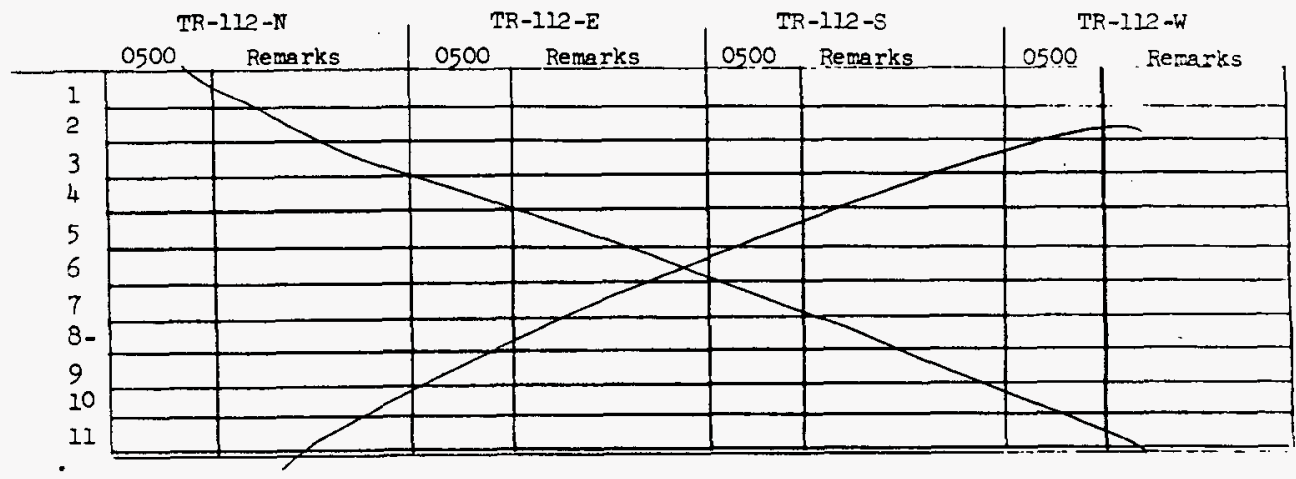

\begin{tabular}{|c|c|}
\hline Pt. & $\begin{array}{l}\text { TR-112-4 } \\
\text { Days }\end{array}$ \\
\hline 1 & 58 \\
\hline 2 & $6 \%$ \\
\hline 3 & 59 \\
\hline 4 & 250 \\
\hline 5 & 160 \\
\hline 6 & 133 \\
\hline 7 & 138 \\
\hline 8 & 164 \\
\hline 9 & 24 \\
\hline 10 & 453 \\
\hline 22 & 123 \\
\hline 12 & 108 \\
\hline 13 & $<$ \\
\hline 14 & 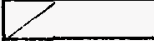 \\
\hline 15 & 2 \\
\hline 16 & 128 \\
\hline 17 & 20 \\
\hline 18 & 119 \\
\hline 19 & $0 / 2$ \\
\hline 20 & 140 \\
\hline $\begin{array}{l}21 \\
22\end{array}$ & 8 \\
\hline 23 & 128 \\
\hline 24 & 76 \\
\hline
\end{tabular}

TR-112-1 TR-112-2 0500
$T R-24 I-E X-2$ 0500

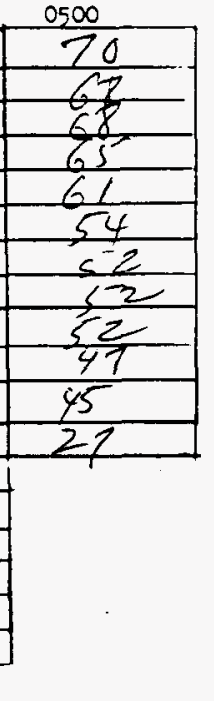

Record on 8-4 Sh1ft

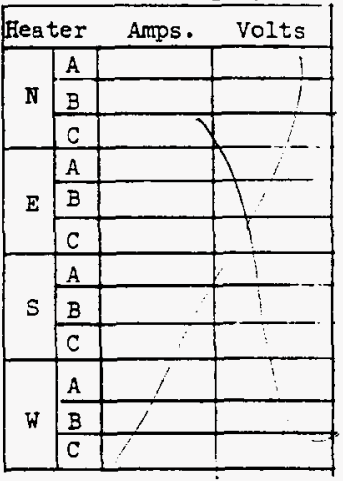

INSTRUCTIONS:

1. F1II in all blanks

2. If data are not avallable, glve reason under Rewarks.

3. If recording instruments, thermocouples, etc. are not functioning properly, report this to supervision.

4. Circle all results that are out of process limits or are otherwse questionable.

5. All suggestions, formal or informal, w11l be given prompt attention. 

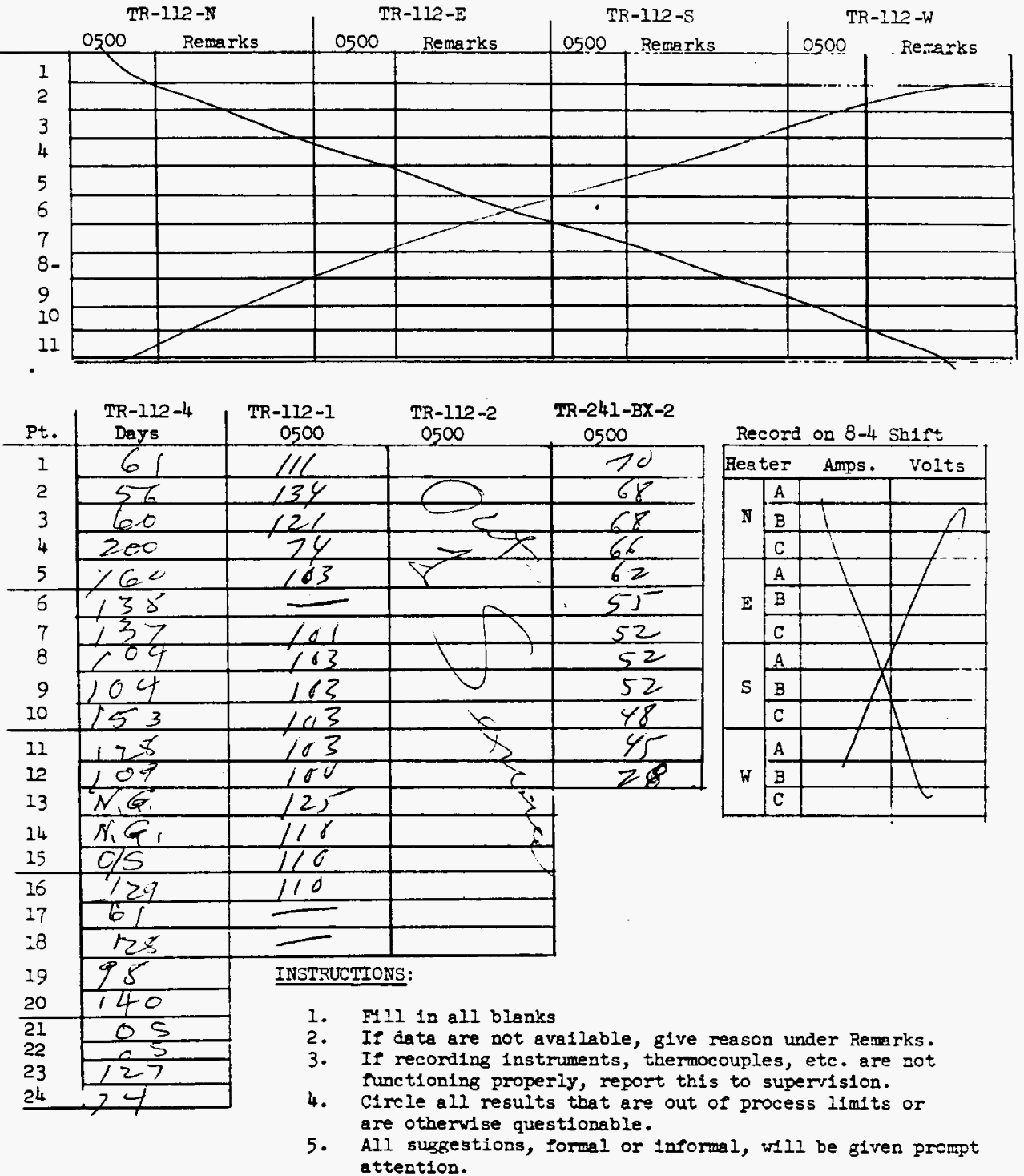


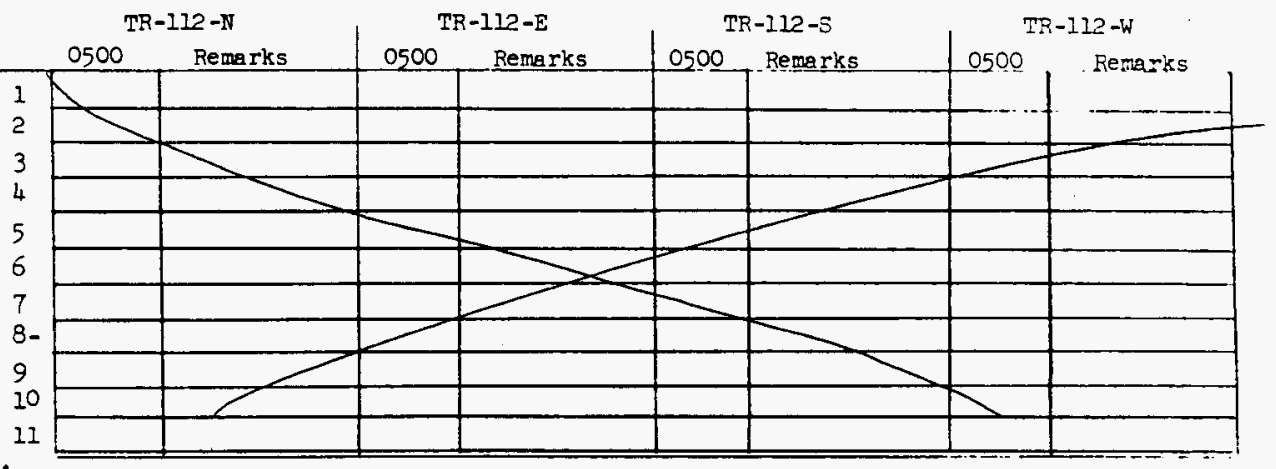

\begin{tabular}{|c|c|}
\hline Pt. & $\begin{array}{l}\text { TR-112-4 } \\
\text { Days }\end{array}$ \\
\hline 1 & 62 \\
\hline 2 & 67 \\
\hline 3 & 62 \\
\hline 4 & 200 \\
\hline 5 & 160 \\
\hline 6 & 139 \\
\hline 7 & 138 \\
\hline 8 & $10 \%$ \\
\hline 9 & 80 \\
\hline 10 & 153 \\
\hline 21 & 129 \\
\hline 12 & $10 \%$ \\
\hline 13 & 000 \\
\hline 14 & 000 \\
\hline 15 & 350 \\
\hline 16 & 129 \\
\hline 17 & -62 \\
\hline 18 & 120 \\
\hline 19 & 96 \\
\hline 20 & 140 \\
\hline 21 & 350 \\
\hline $\begin{array}{l}22 \\
23\end{array}$ & 136150 \\
\hline 24 & 80 \\
\hline & $10-30-74$ \\
\hline
\end{tabular}

TR-112-1 TR-112-2 0500
TR-241-BX-2 0500 $7 \%$

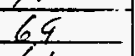
66 62 55 52 52 52 48 45 45
26 $10-30-7 y$ Rev $O$ 


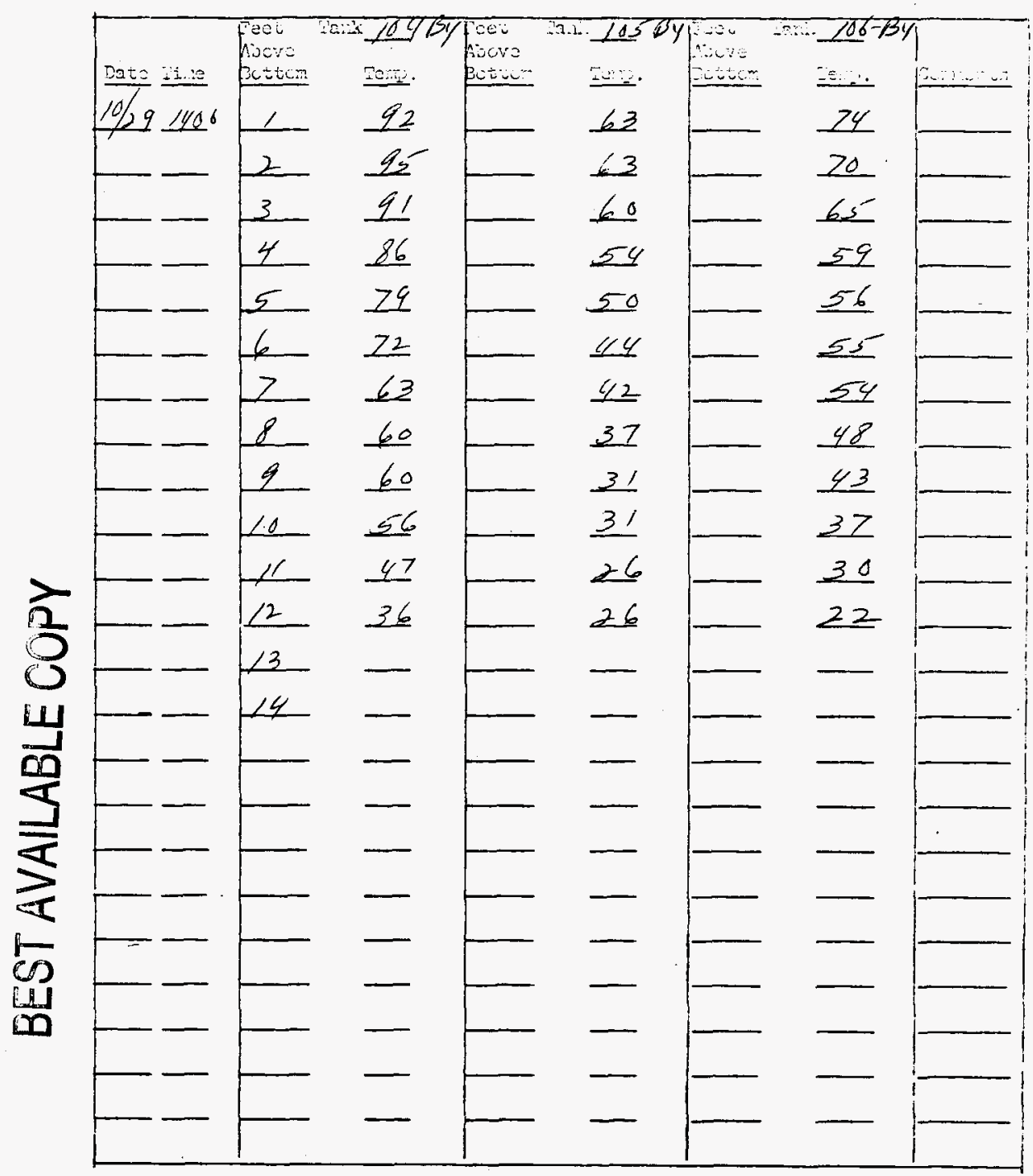

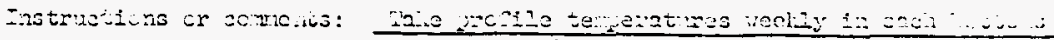

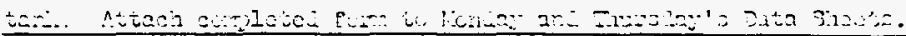


2010

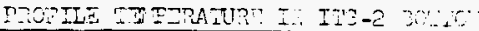

$-4 . j \mathrm{SC}$

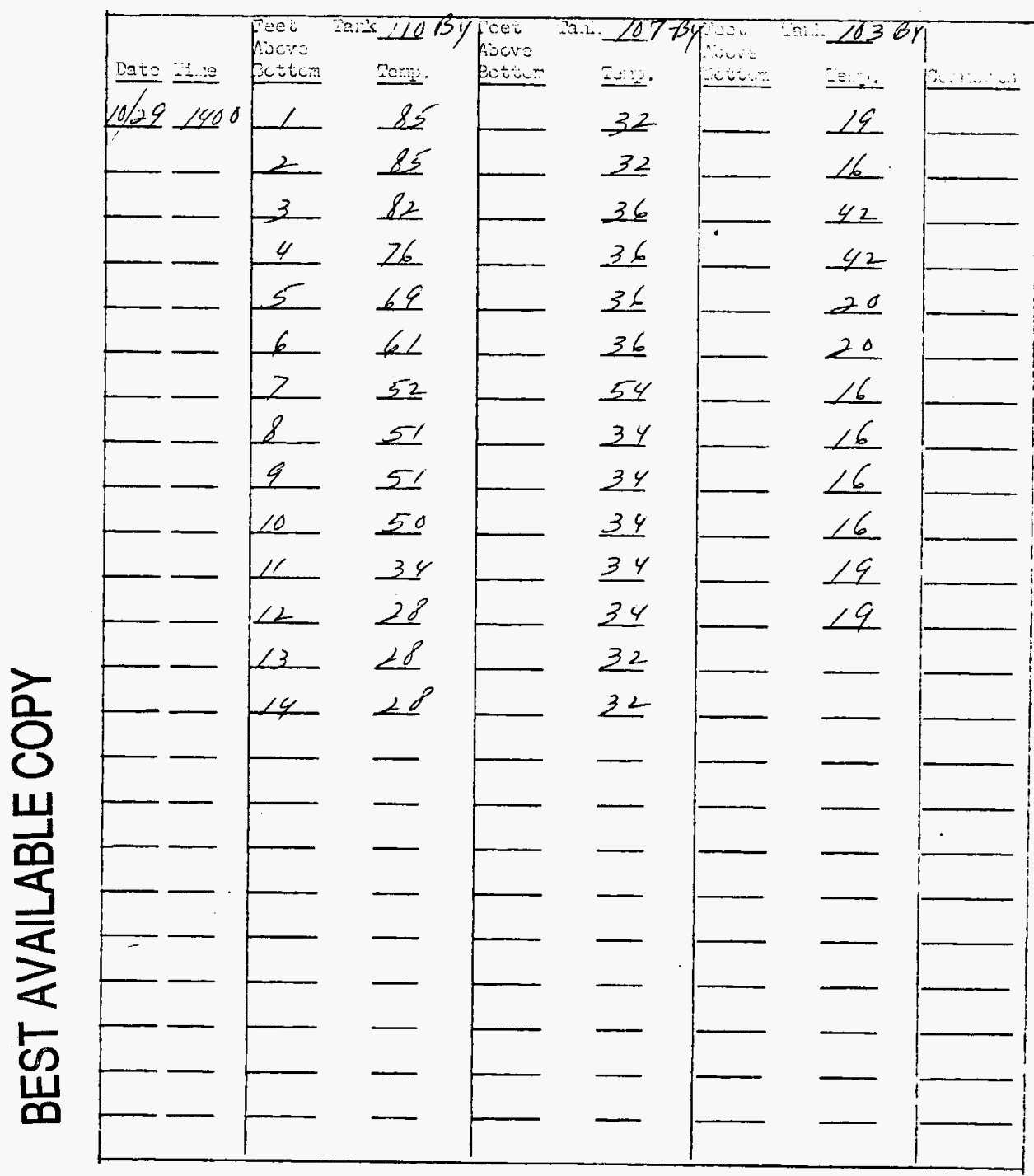

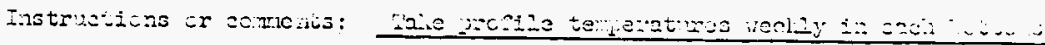

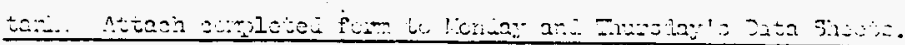




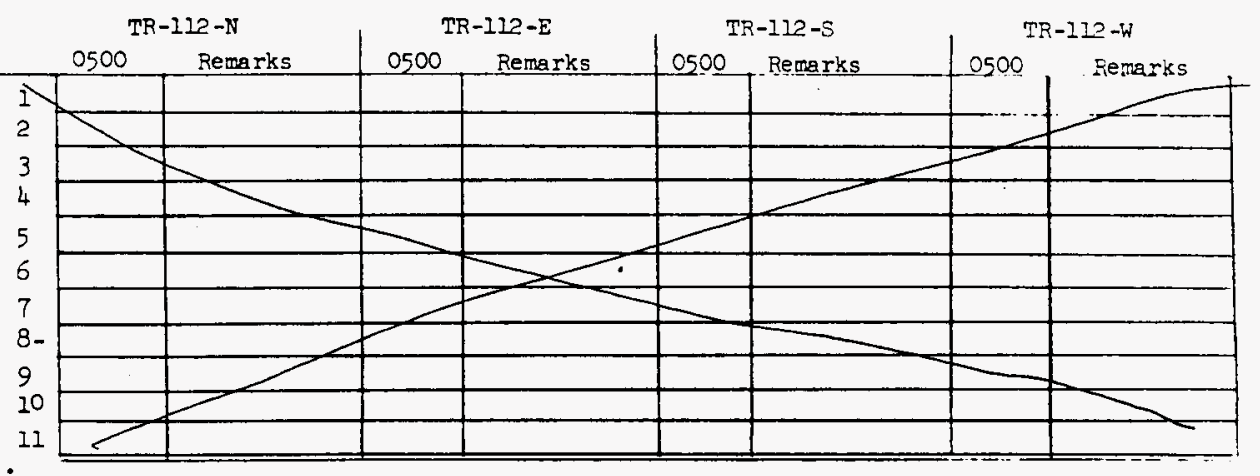

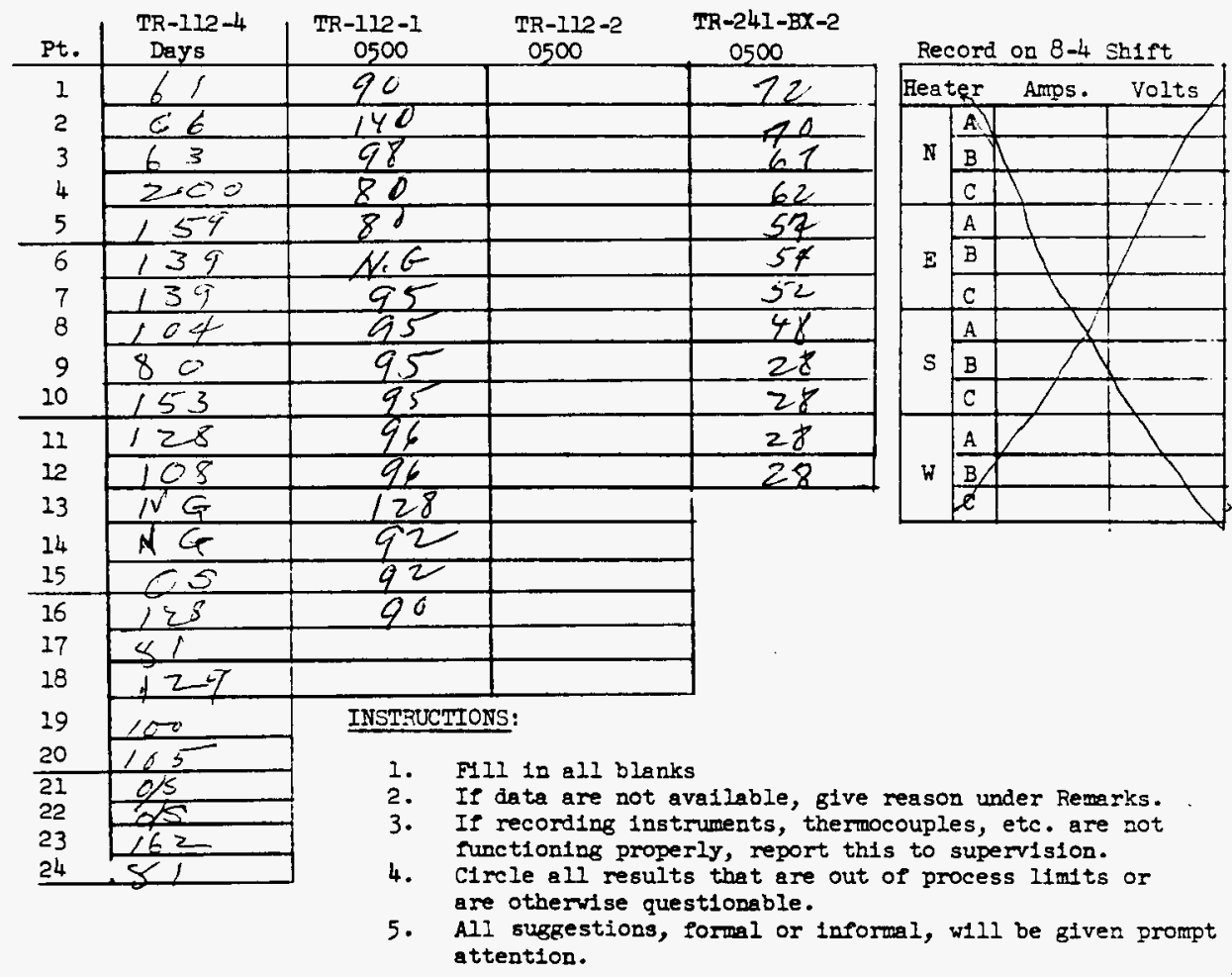


IIS -2 TEMPERATURES
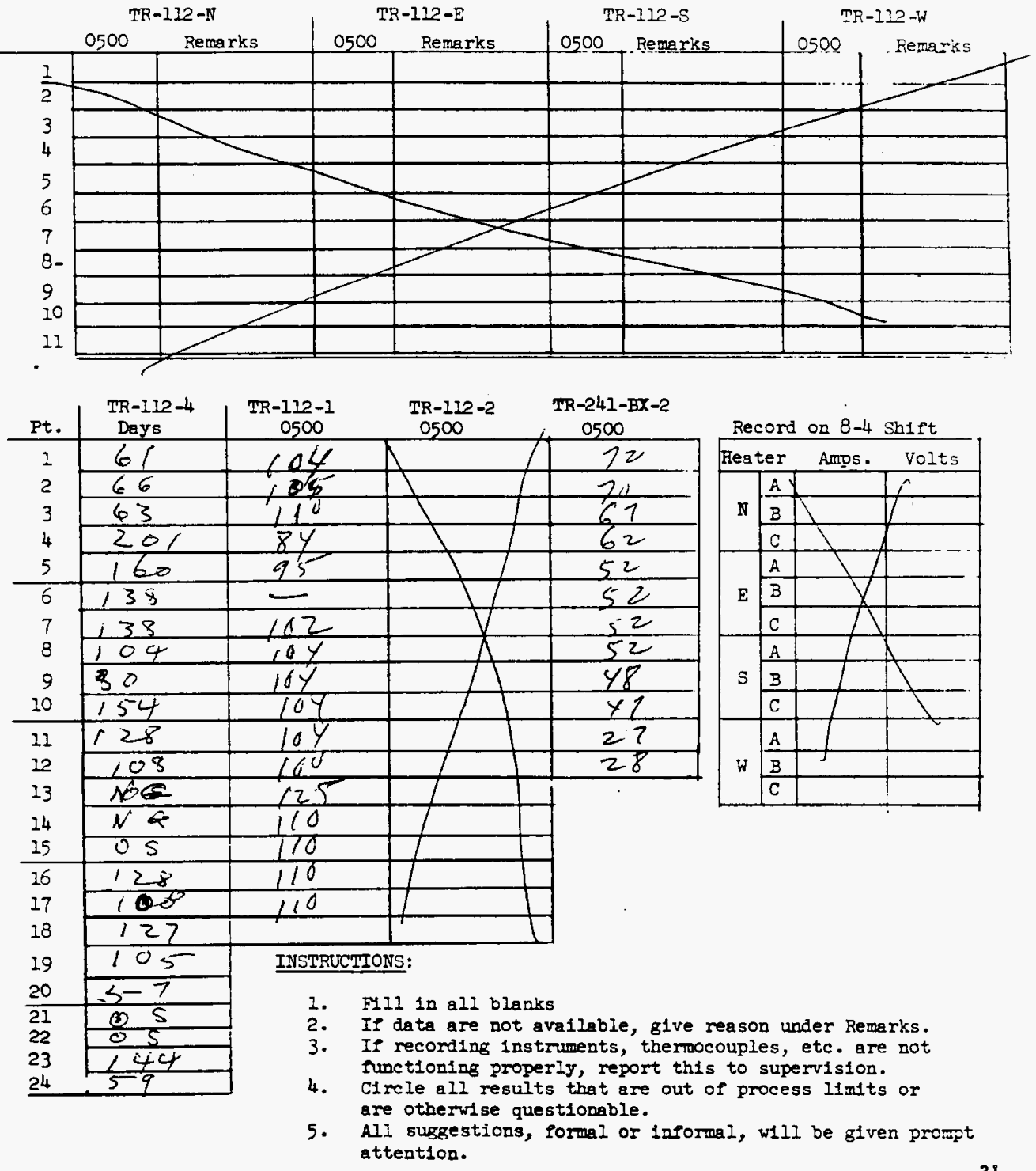
ITS-2 TEMPERATURES
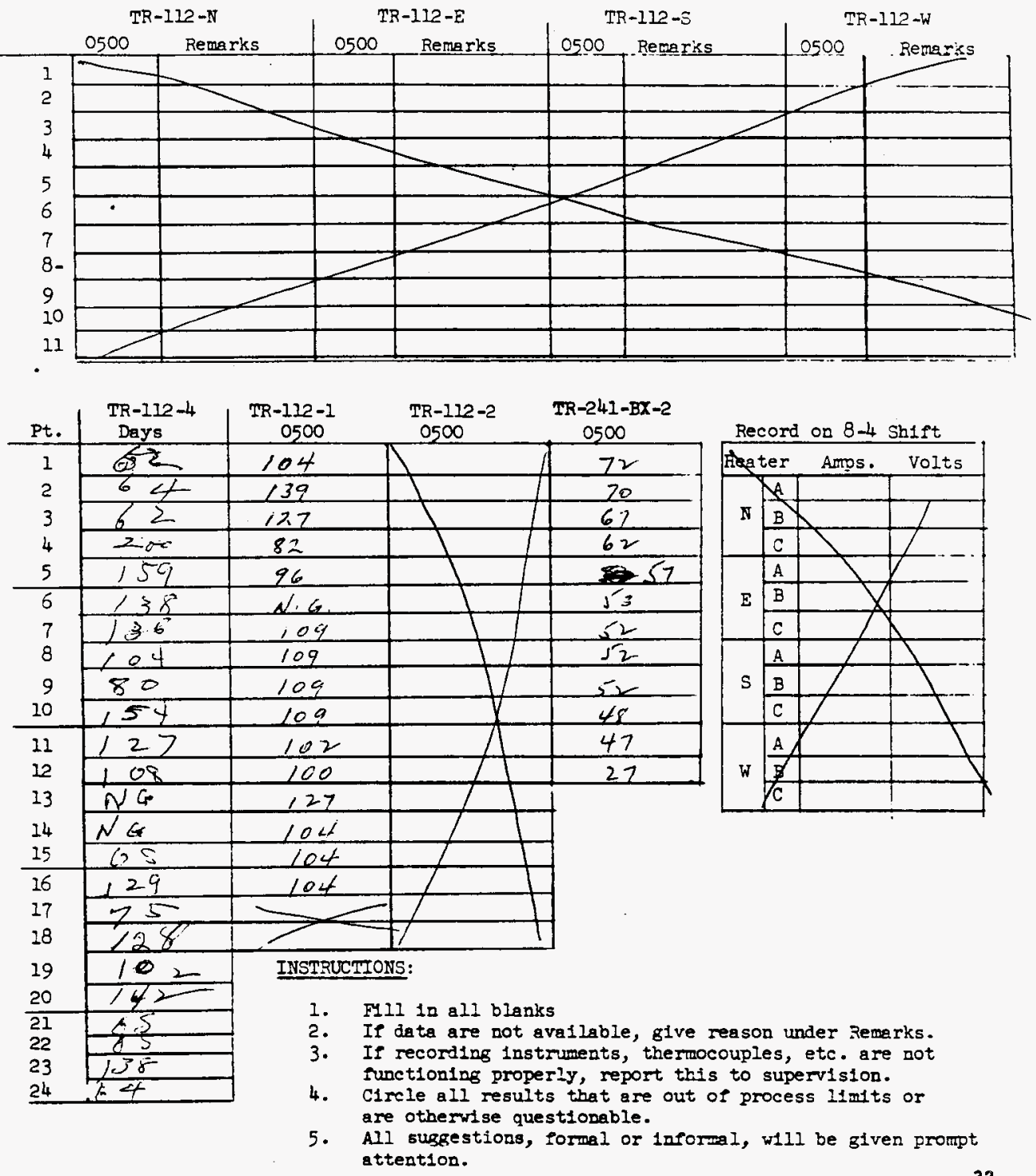

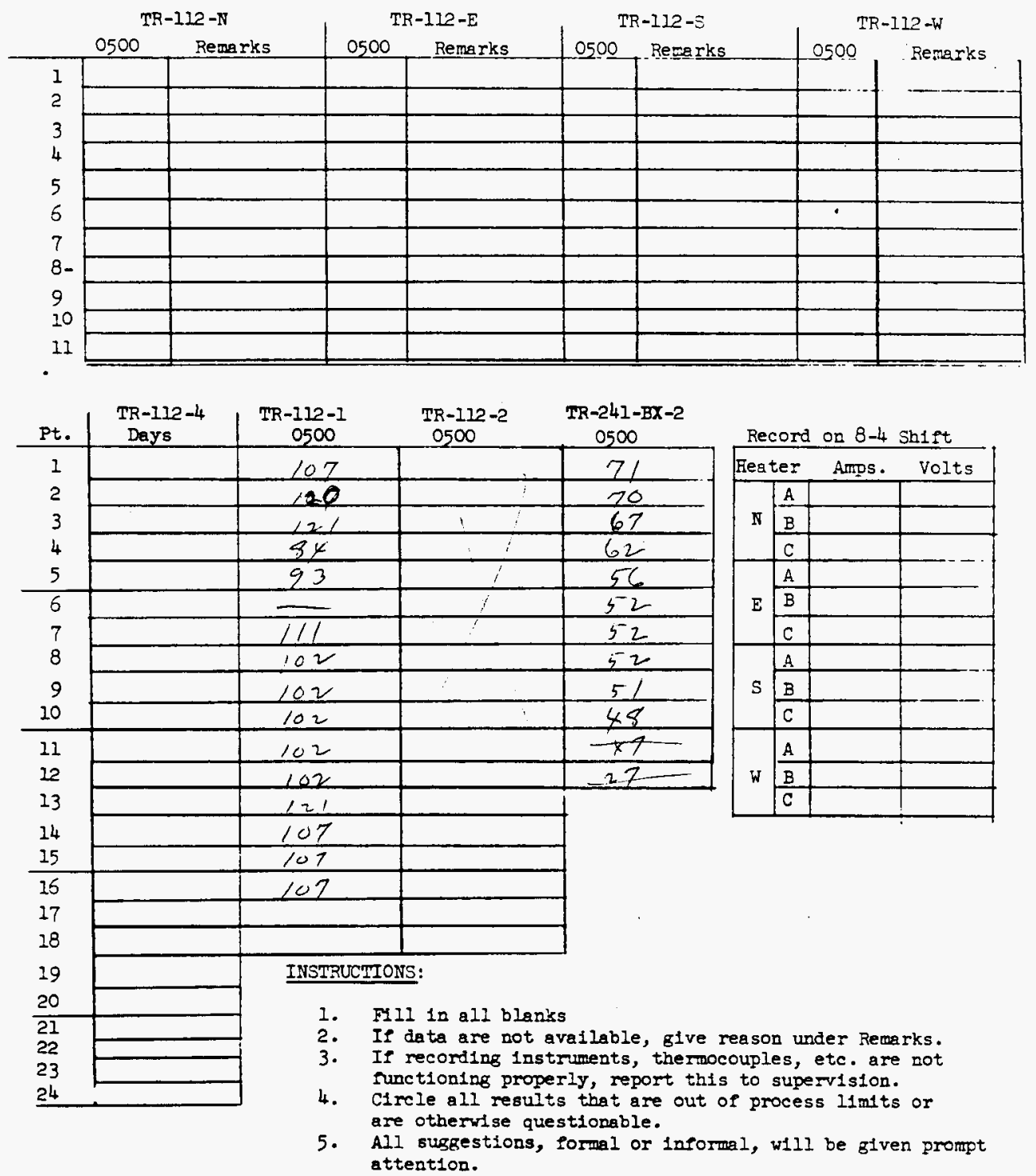

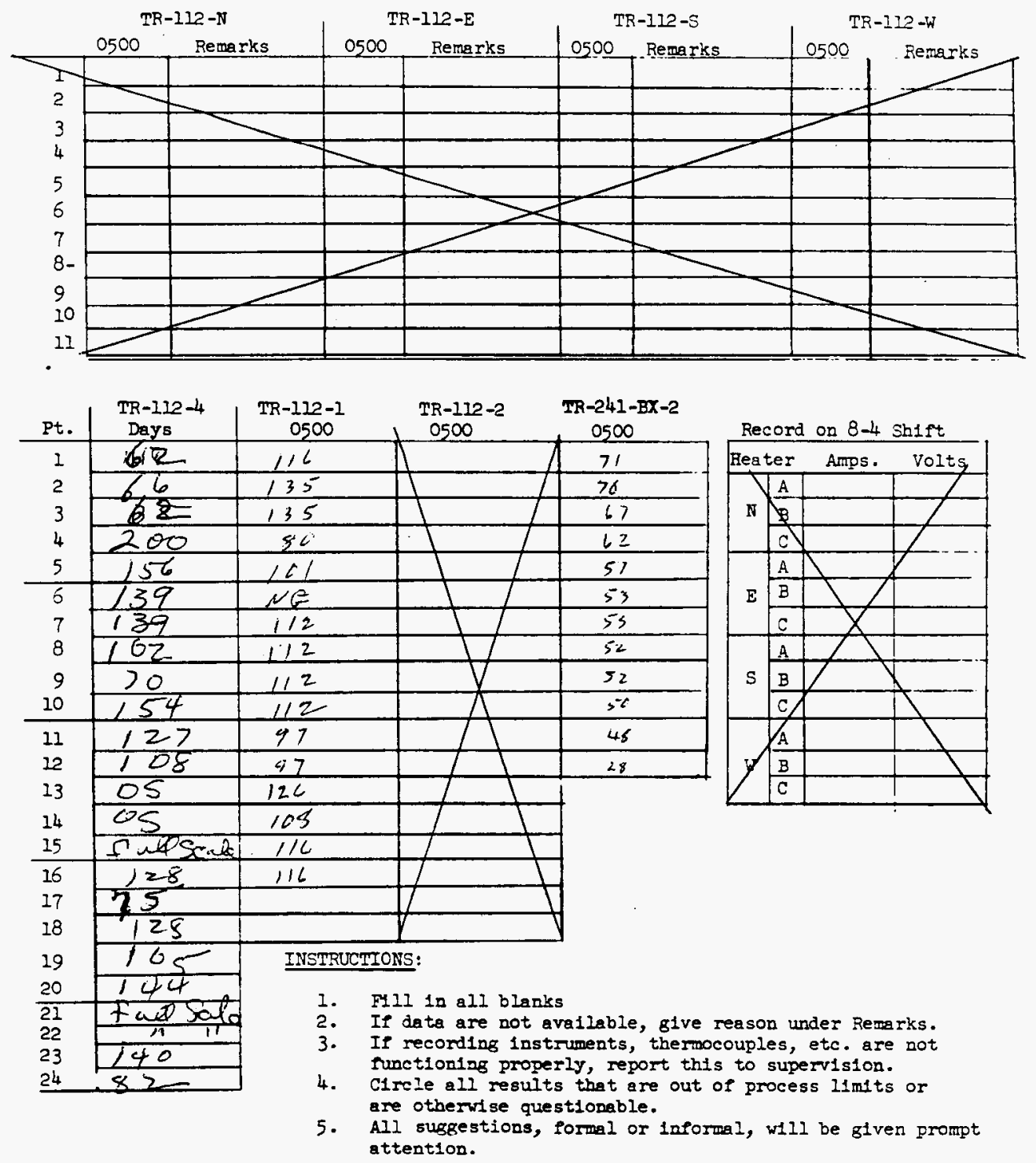
ITS -2 TTEMPERATURES

Date: $10-2-4=74$
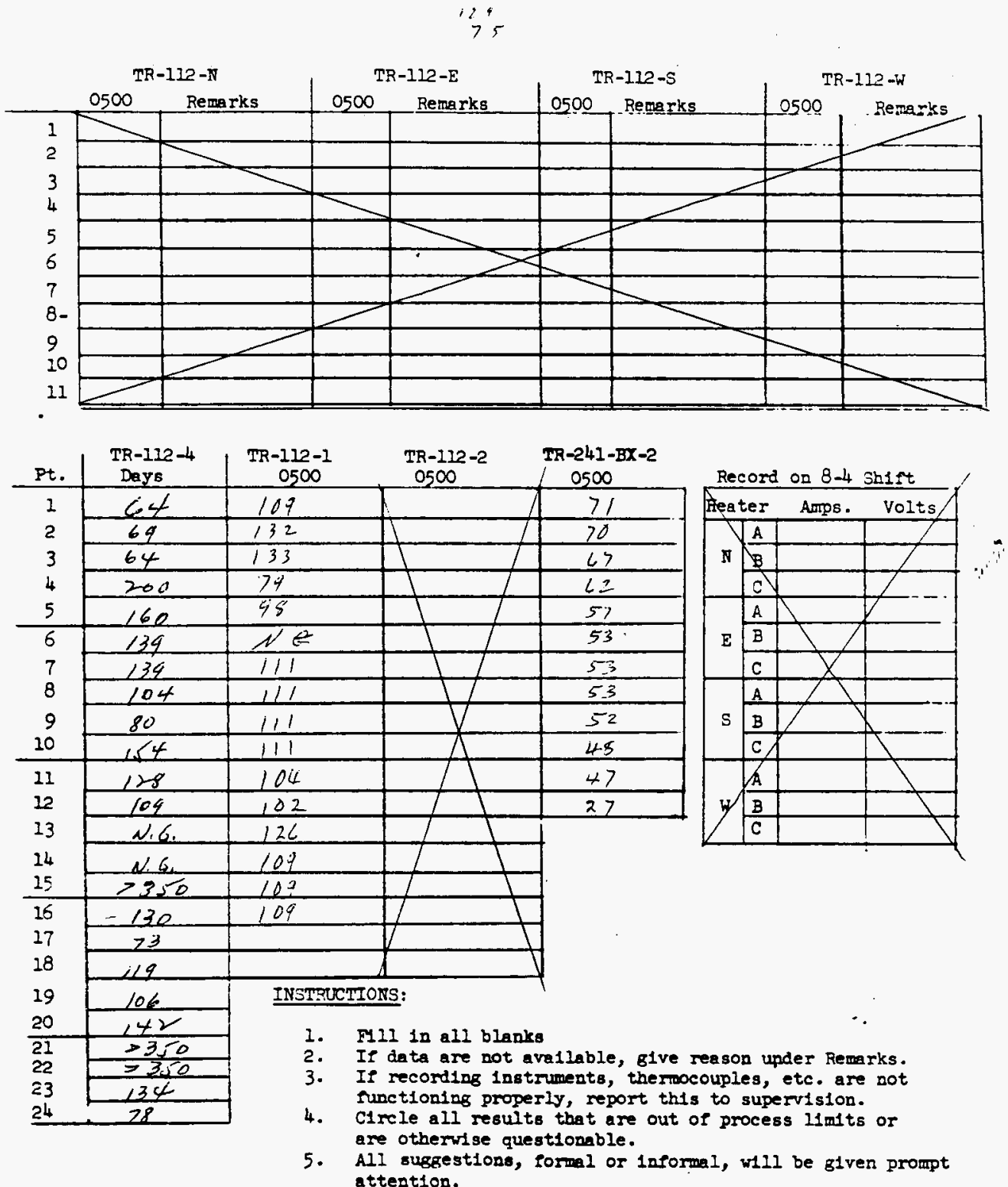

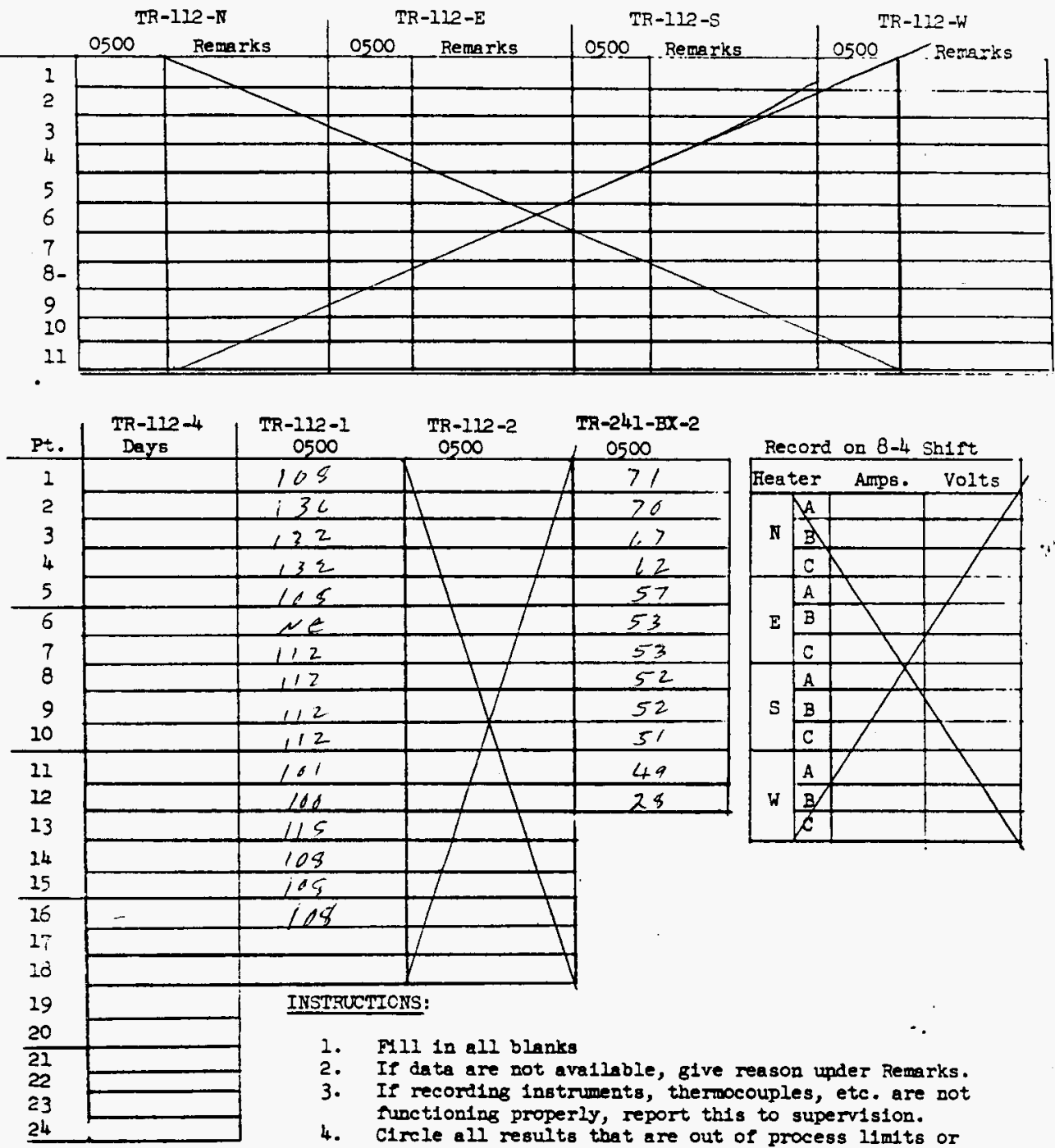

$\begin{array}{ccc}\text { TR-112-1 } & \text { TR-112-2 } & \text { TR-241-BX-2 } \\ 0500 & 0500 & 0500\end{array}$

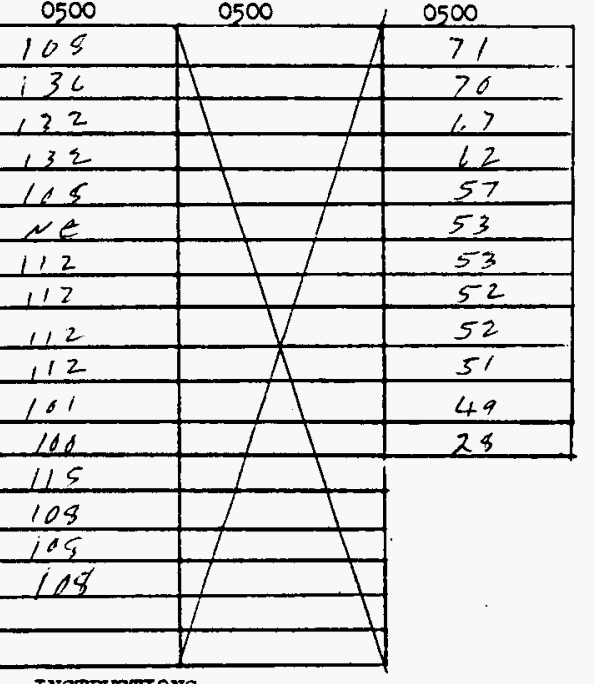

INSTRUCTICNS:

1. F11 in all blanks

2. If data are not avaliable, give reason upder Remarks.

3. If recording instruments, therwocouples, etc, are not functioning properly, report this to supervision.

4. Circle all results thet are out of process limits or are otberwlse questionable.

5. All suggestiona, formal or 1nformal, w1l be given prompt attent10n.

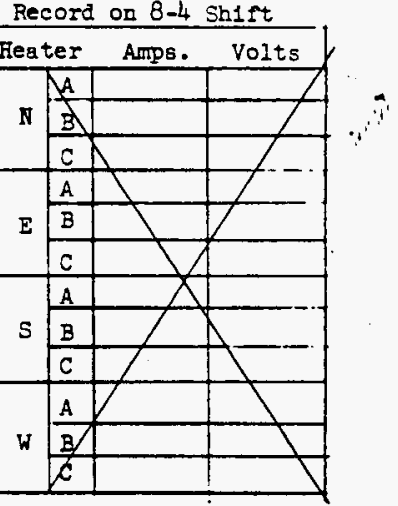


ITS -2 TEMPERATURES

WHC-SD-WM-DP-208

Date: $10-22-74$

TR-112-N

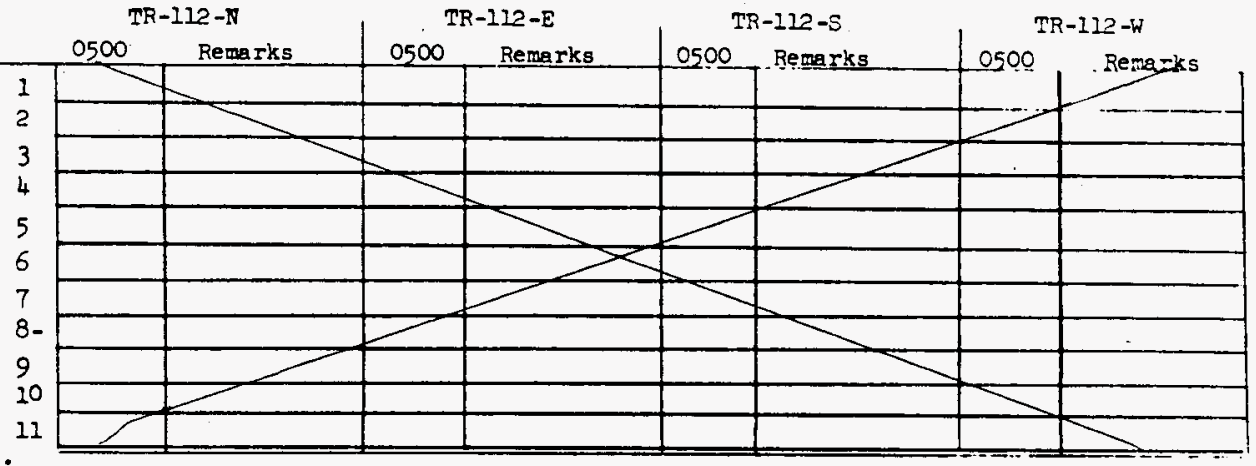

\begin{tabular}{|c|c|}
\hline Pt. & $\begin{array}{c}T R-112-4 \\
\text { Deys }\end{array}$ \\
\hline \multirow{5}{*}{$\begin{array}{l}1 \\
2 \\
3 \\
4 \\
5\end{array}$} & 66 \\
\hline & 72 \\
\hline & 66 \\
\hline & 201 \\
\hline & 160 \\
\hline \multirow{3}{*}{$\begin{array}{l}6 \\
7 \\
8\end{array}$} & 139 \\
\hline & 139 \\
\hline & 103 \\
\hline \multirow{2}{*}{$\begin{array}{r}9 \\
10 \\
\end{array}$} & 81 \\
\hline & 155 \\
\hline 12 & 128 \\
\hline 12 & 109 \\
\hline 13 & $\leq 0$ \\
\hline 14 & 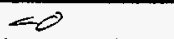 \\
\hline 15 & $>350$ \\
\hline 16 & $\Rightarrow 130$ \\
\hline 17 & 72 \\
\hline 18 & 119 \\
\hline 19 & 106 \\
\hline 20 & 144 \\
\hline 21 & 3350 \\
\hline 22 & $\geq 350$ \\
\hline 23 & 133 \\
\hline 24 & 78 \\
\hline
\end{tabular}

TR-112-1 TR-112-2 TR-241-BX-2 0500 106 137 132

80
45 NE 111

111

111
102 103 135 106 106

\section{INSTRUCIIONS:}
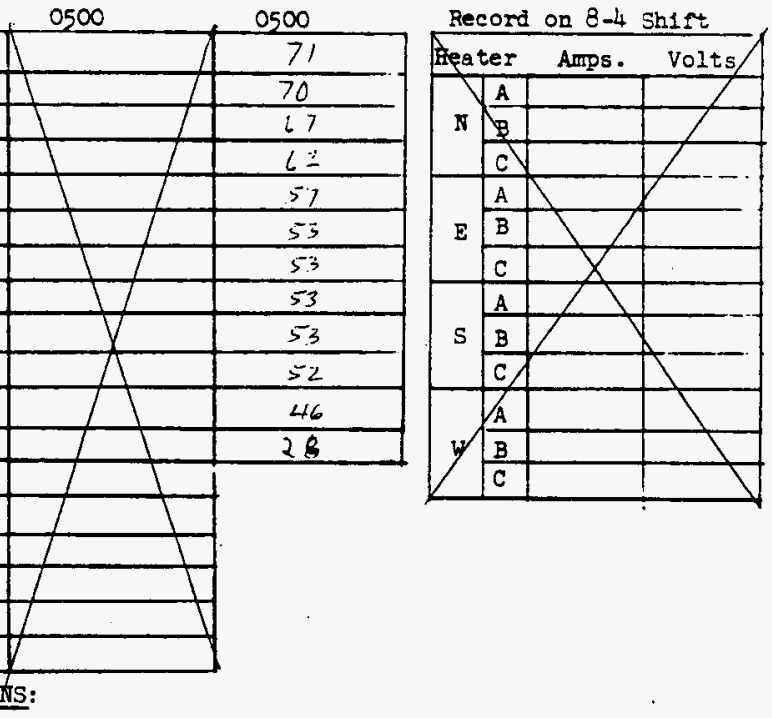

1. F11 in all blanks

2. If data are not avalable, give reason under Remarks.

3. If recording Instruments, thermocouples, etc. are not functioning properly, report this to supervision.

4. C1rcle all results that ere out of process $11 \mathrm{ntts}$ or are otherwise questionable.

5. All suggestions, formal or informal, wil be given prompt attent1on. 


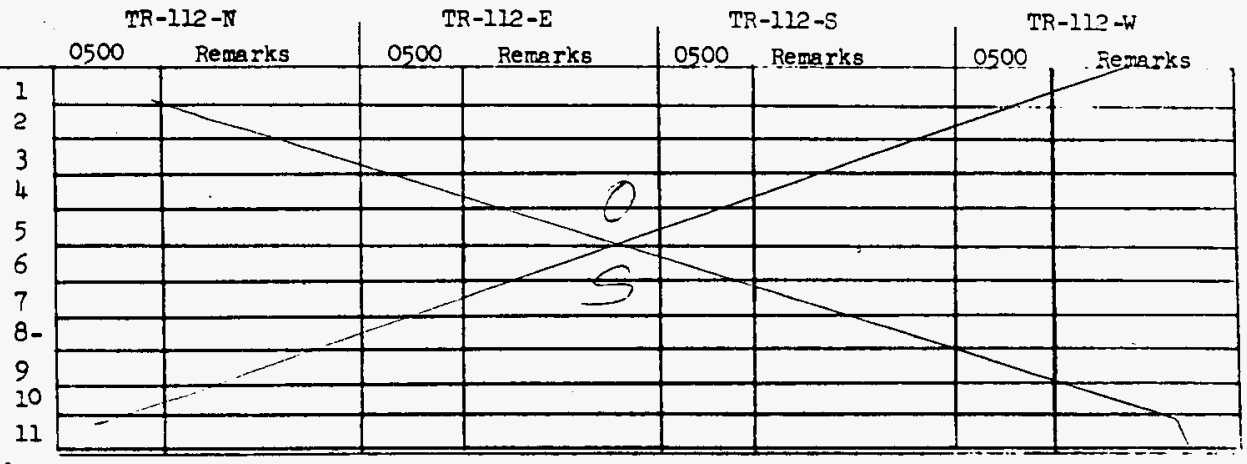

\begin{tabular}{|c|c|c|c|c|}
\hline Pt. & $\begin{array}{l}\text { TR-112-4 } \\
\text { Days }\end{array}$ & $\begin{array}{c}\text { TR-112-1 } \\
0500\end{array}$ & $\begin{array}{c}\text { TR-112-2 } \\
0500\end{array}$ & $\begin{array}{l}\mathrm{TR}-241-\mathrm{BX}-2 \\
\quad 0500\end{array}$ \\
\hline 1 & 56 & 107 & & 71 \\
\hline 2 & 74 & 137 & 7 & 70 \\
\hline 3 & 52 & 123 & I & 67 \\
\hline 4 & 201 & 80 & 1 & 62 \\
\hline 5 & 161 & 85 & 7 & 57 \\
\hline 6 & 140 & $N E$ & $T$ & 53 \\
\hline 7 & 139 & 105 & 1 & 52 \\
\hline 8 & 103 & 105 & 21 & 52 \\
\hline 9 & 81 & 105 & V & 52 \\
\hline 10 & 154 & 105 & $\pi$ & 51 \\
\hline 11 & 128 & 100 & 5 & 43 \\
\hline 12 & 110 & 98 & 1 & 21 \\
\hline 13 & $\leq 0$ & 127 & 1 & \\
\hline 14 & $\leq 0$ & 96 & 1 & \\
\hline 15 & 2350 & 96 & 1 & \\
\hline 16 & +30 & 107 & I & \\
\hline 17 & 68 & & & \\
\hline 18 & 119 & & & \\
\hline 19 & 106 & \multicolumn{2}{|c|}{ INSTRUCTIONS: } & \\
\hline
\end{tabular}

Record on 8-4 Shift

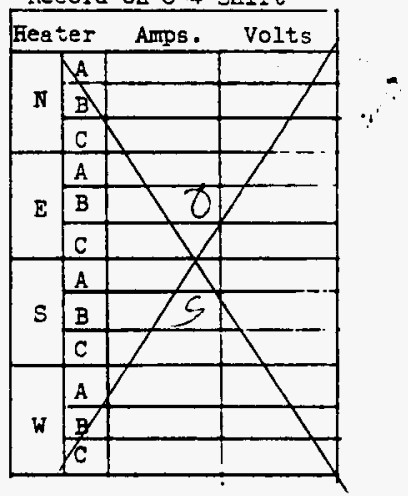

1. F111 in all blanks

2. If data are not avallable, glve reason under Remarks.

3. If recording instruments, thermocouples, etc. are not runctioniag properly, report this to supervision.

4. C1rele all results thet are out of process $11 m 1$ ts or are otherwlse questionable.

5. All susgestions, formal or informal, wlll be given prompt attention. 
Date: $\because-20.76$
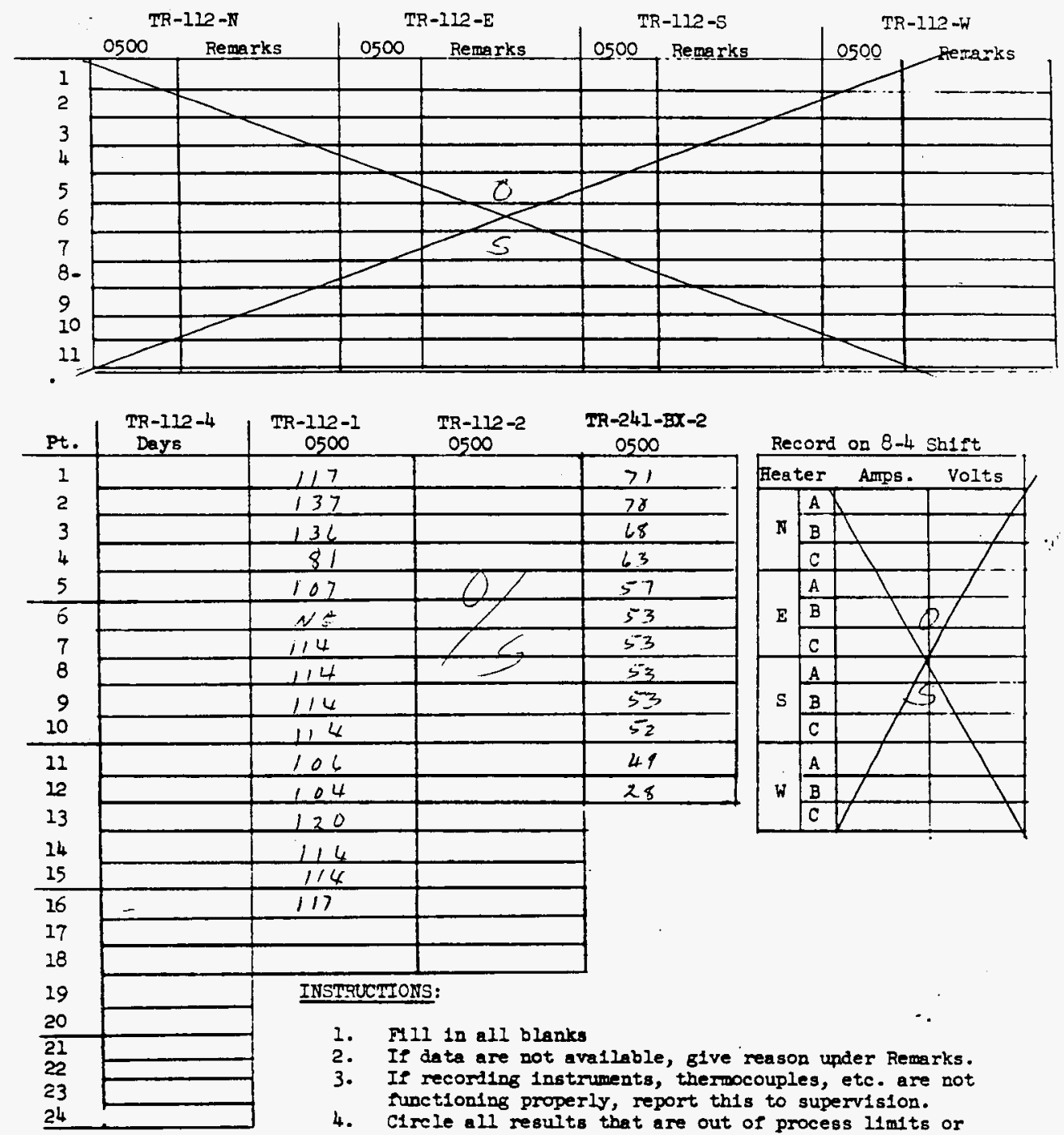

\begin{tabular}{c}
$T R-112-I$ \\
0500 \\
\hline
\end{tabular}

\section{TR-112-2}

117

137

136

81

107

N

i, 4

1,4

114

106

104

120

114

117

INSTRUCTONS:

1. F11 1n aIl blanks

2. If date are not avalleble, give reason under Remarks.

3. If recording 1nstruments, therwocouples, etc. are not runctioning properly, report this to supervision.

4. Circle all resuits that are out of process ilmits or are otherwlse questionable.

5. All susgestions, formal or informel, w1ll be glven prompt attention.

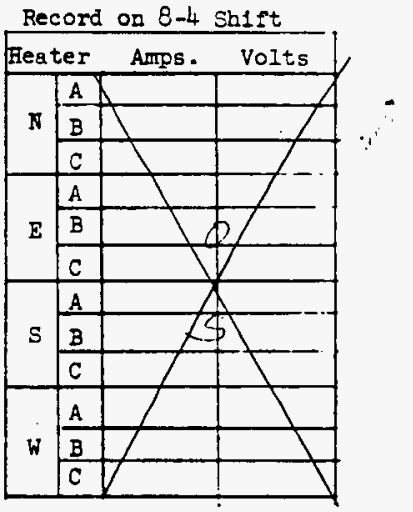



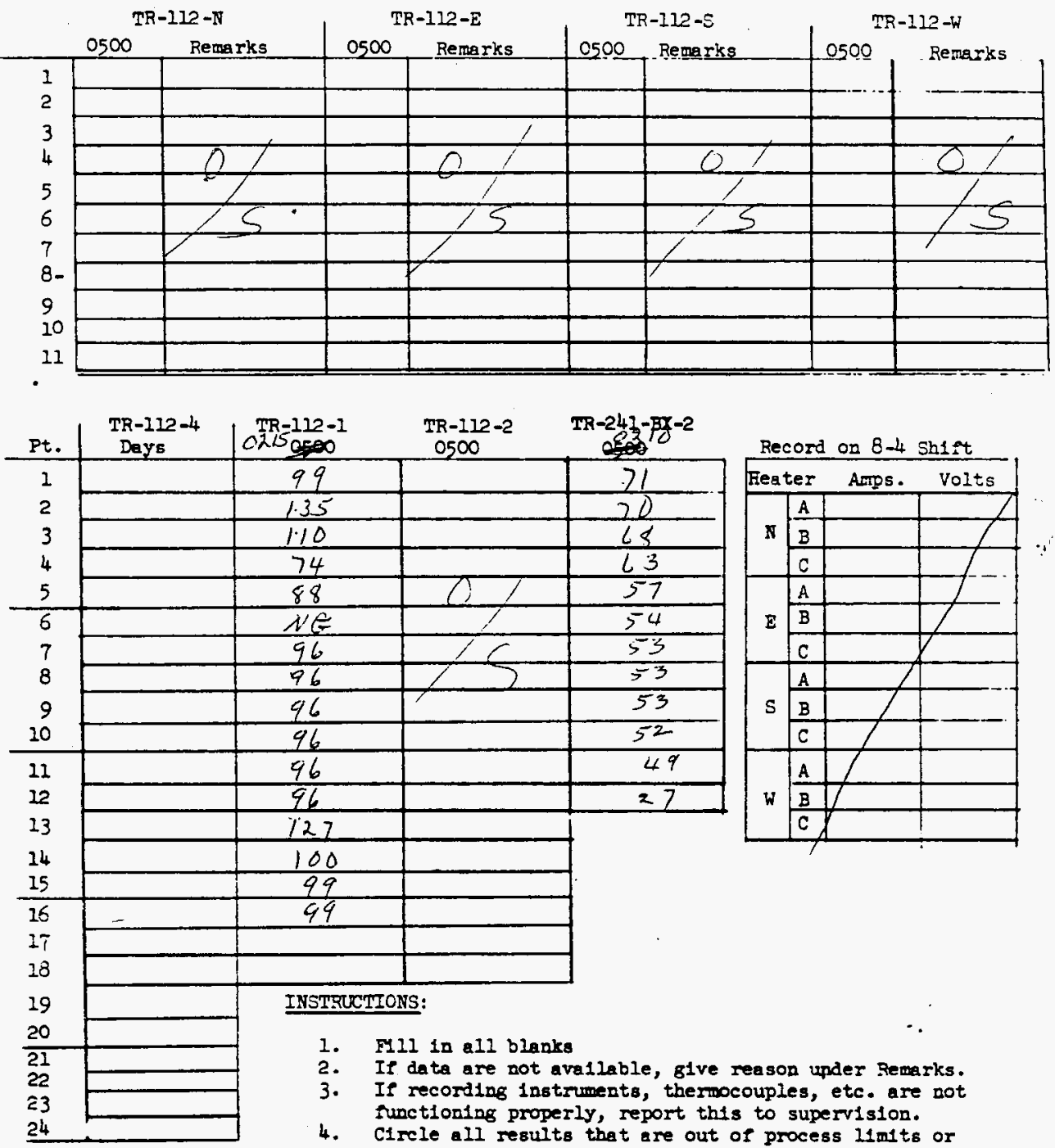

INSTRUCTIONS:

1. F111 in all blanks

2. If data are not avallable, give reason upder Remarks.

3. If recording instruments, thermocouples, etc. are not runctioning properly, report this to supervision.

4. Circle all results that are out of process limlts or are otherwise questionable.

5. All suggestions, formal or 1nformal, w1ll be given prompt attention. 

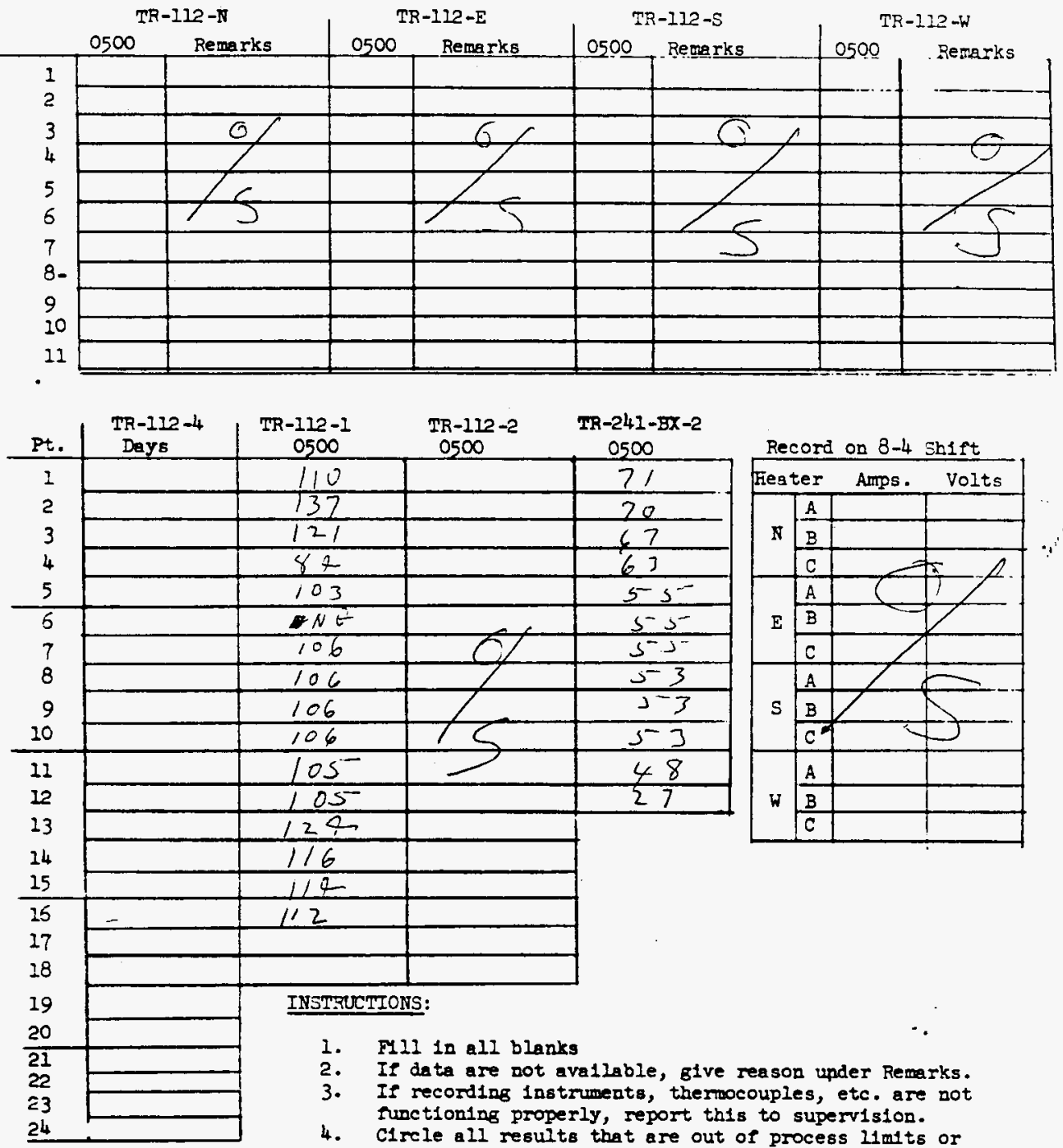

TR-112-1 TR-112-2 TR-241-3X-2

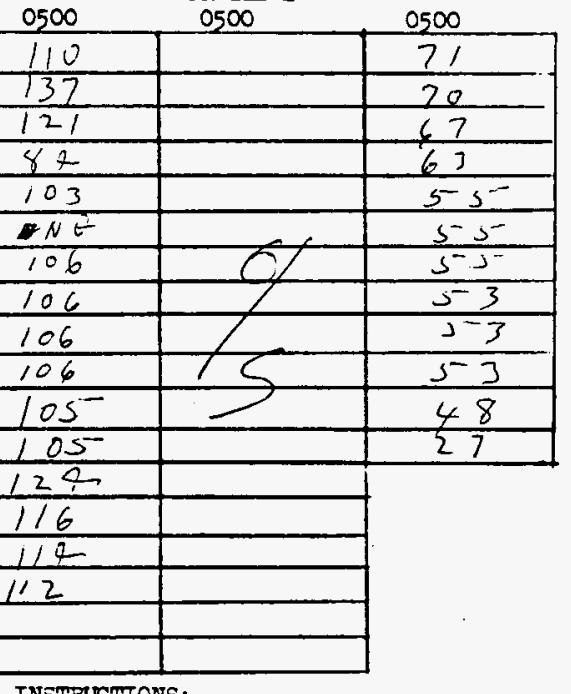

INSTRUCTIONS:

1. F111 in alI blanks

2. If data are not avallable, give reason under Remarks.

3. If recordins instrumeats, thermocouples, etc. are not runctioning properly, report this to supervision.

4. Circle all results that are out of process 11 mits or are otherwlse questionable.

5. All suggestions, formal or informal, will be given prompt attent1on. 
Date: $10-17-74$
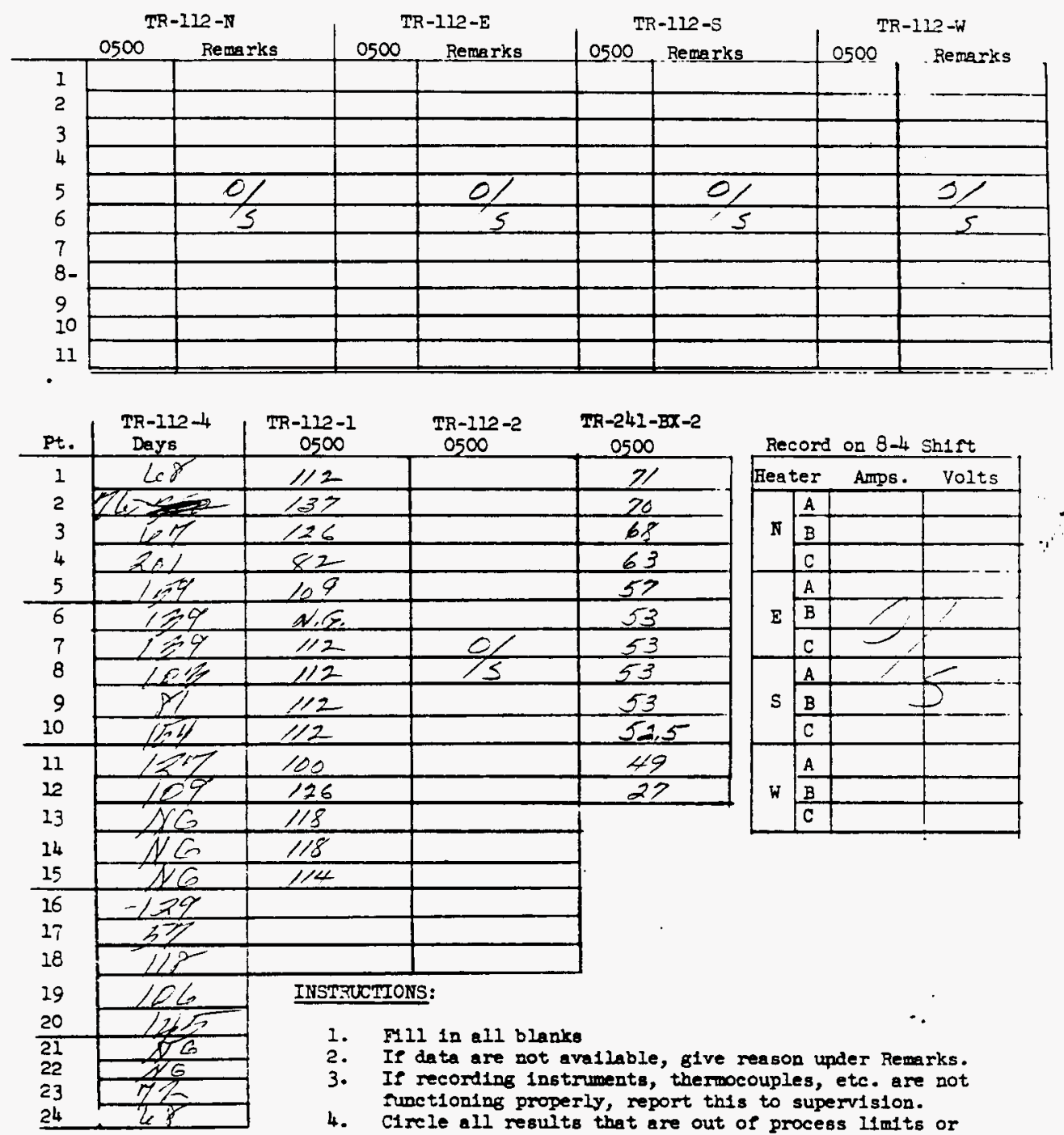

$\begin{array}{ccc}\text { TR- } 112-1 & \text { TR-112-2 } & \text { TR-241-BX-2 }\end{array}$

\begin{tabular}{|c|c|c|}
\hline 0500 & 0500 & 0500 \\
\hline 112 & & 71 \\
\hline 37 & & 70 \\
\hline 126 & & 68 \\
\hline 82 & & 63 \\
\hline 109 & & 57 \\
\hline 112 & & 53 \\
\hline 112 & $0 / 2$ & 53 \\
\hline 112 & 15 & 53 \\
\hline 112 & & 53 \\
\hline 100 & & 515 \\
\hline 126 & & 49 \\
\hline 118 & & 27 \\
\hline 118 & & \\
\hline
\end{tabular}

Record on 8-4 Shift

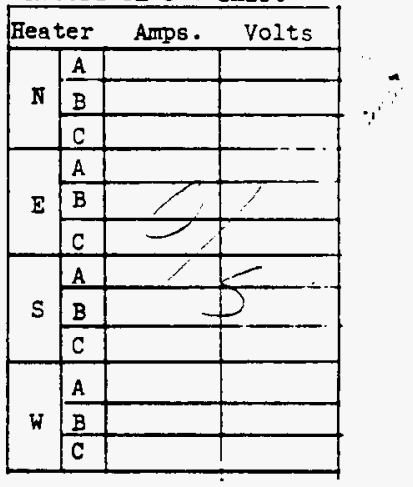

$1 / 4$

INSTRUCTIONS:

1. F11 in all blanks

2. If data are not avallable, give reason upder Remsks.

3. If recording instruments, thernocouples, etc. are not

runctioning properly, report this to supervision.

4. Circle all results that are out of process ilmits or are otherwise questionable.

5. All suggestions, formal or 1nformal, will be given prompt attent1on. 

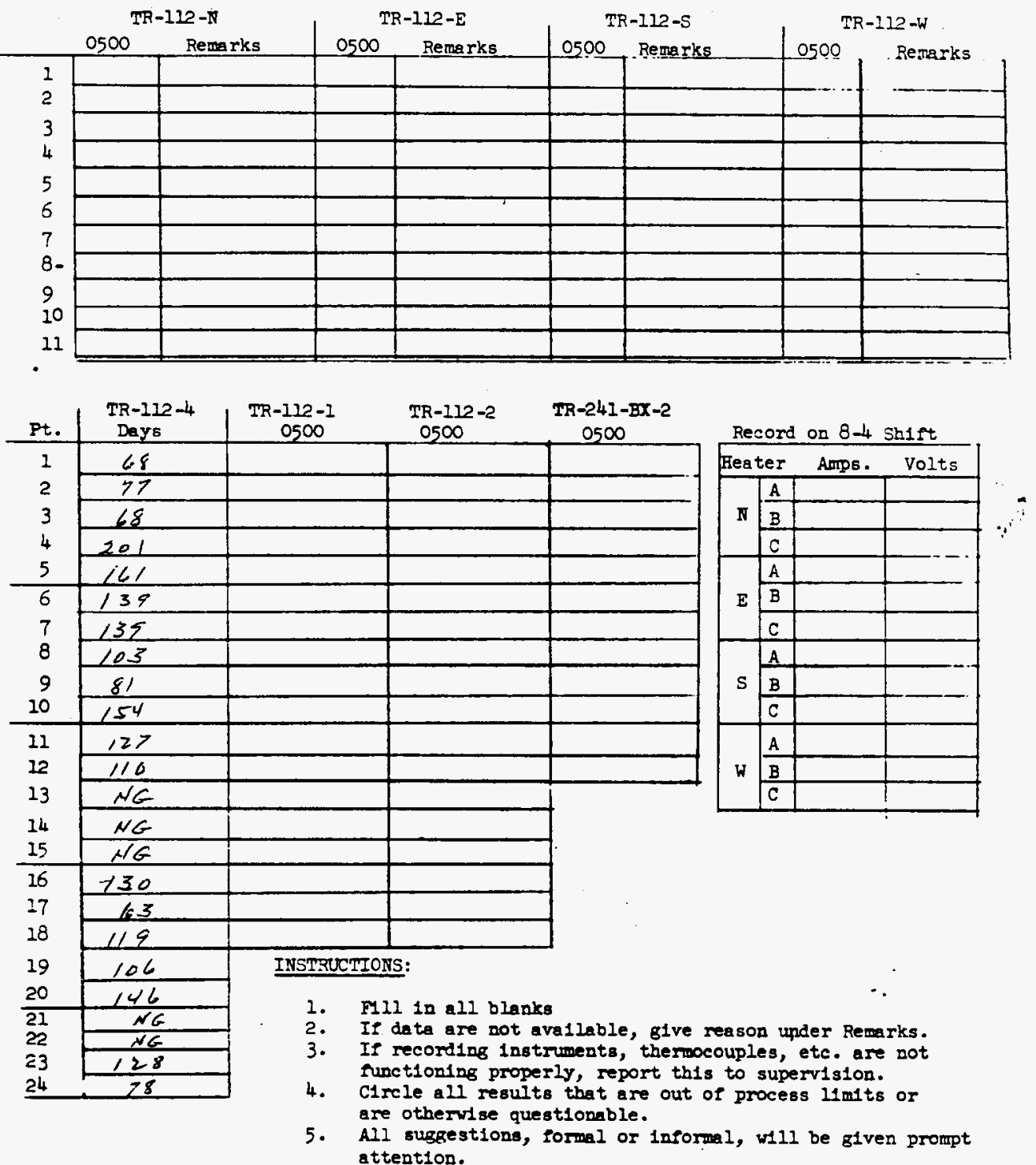


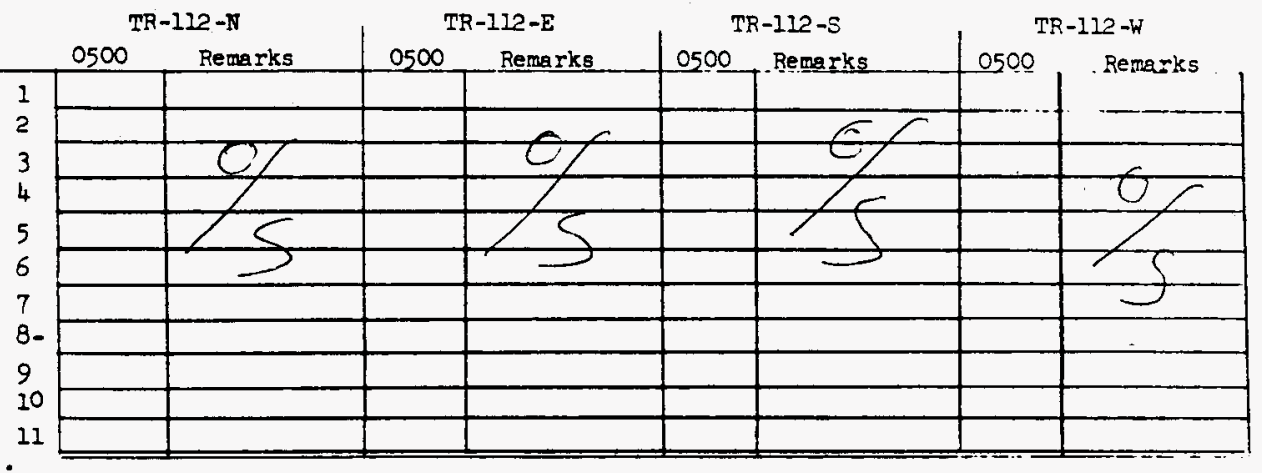

\begin{tabular}{|c|c|}
\hline Pt. & $\begin{array}{c}T R-112-4 \\
\text { Deys }\end{array}$ \\
\hline \multirow{5}{*}{$\begin{array}{l}1 \\
2 \\
3 \\
4 \\
5\end{array}$} & 69 \\
\hline & 82 \\
\hline & 6.8 \\
\hline & 201 \\
\hline & 161 \\
\hline \multirow{5}{*}{$\begin{array}{r}6 \\
7 \\
8 \\
9 \\
10 \\
\end{array}$} & 140 \\
\hline & 140 \\
\hline & 103 \\
\hline & 82 \\
\hline & 154 \\
\hline \multirow{5}{*}{$\begin{array}{l}11 \\
12 \\
13 \\
14 \\
15\end{array}$} & 127 \\
\hline & 110 \\
\hline & $N G$ \\
\hline & NG \\
\hline & $N G$ \\
\hline \multirow{3}{*}{$\begin{array}{l}16 \\
17 \\
18\end{array}$} & 130 \\
\hline & $65^{-}$ \\
\hline & 119 \\
\hline \multirow{2}{*}{$\begin{array}{l}19 \\
20\end{array}$} & 106 \\
\hline & 146 \\
\hline \multirow{3}{*}{$\begin{array}{l}21 \\
22 \\
23 \\
24 \\
\end{array}$} & $\frac{N G}{N G}$ \\
\hline & 130 \\
\hline & 78 \\
\hline
\end{tabular}

TR-112-2 0500 $1 / 5$

134

128

$\frac{128}{84}$

105

$N G$

110

110

$1 / 0$

110

102

103

125

$11 x$

118

114

INSTRUCTIONS:

1. Fill in all blanks

2. If data are not avallable, give reason under Remarks.

3. If recording instruments, therrocouples, etc. are not functioning properly, report this to supervision.

4. Circle all results that are out of process 11 mits or are otherwise questionable.

5. All susgestions, formal or 1nformal, will be given prompt attention.
Record on 8-4 Shift

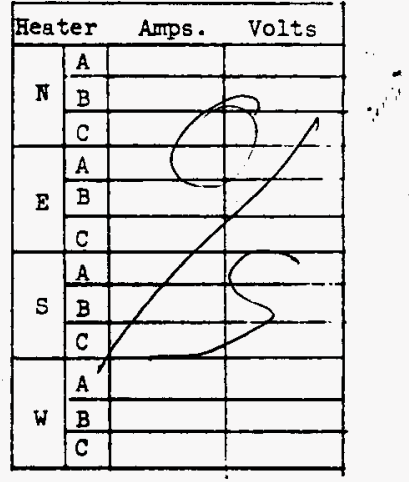




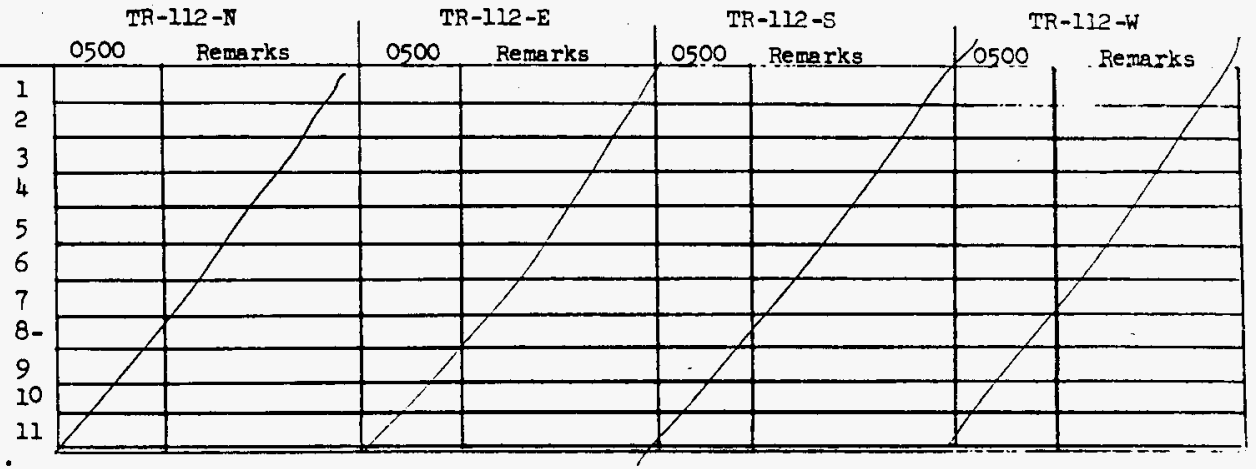

\begin{tabular}{|c|c|}
\hline Pt. & $\begin{array}{c}\text { TR-112-4 } \\
\text { Days }\end{array}$ \\
\hline \multirow{5}{*}{$\begin{array}{l}1 \\
2 \\
3 \\
4 \\
5\end{array}$} & 6.9 \\
\hline & 78 \\
\hline & 68 \\
\hline & 201 \\
\hline & 161 \\
\hline \multirow{5}{*}{$\begin{array}{r}6 \\
7 \\
8 \\
9 \\
10 \\
\end{array}$} & 139 \\
\hline & 139 \\
\hline & 102 \\
\hline & 82 \\
\hline & 154 \\
\hline \multirow{5}{*}{$\begin{array}{l}11 \\
12 \\
13 \\
14 \\
15\end{array}$} & 127 \\
\hline & 110 \\
\hline & $N \in$ \\
\hline & NE \\
\hline & $N G$ \\
\hline \multirow{5}{*}{$\begin{array}{l}16 \\
17 \\
18 \\
19 \\
20\end{array}$} & 130 \\
\hline & 63 \\
\hline & 119 \\
\hline & 106 \\
\hline & 145 \\
\hline \multirow{3}{*}{$\begin{array}{l}21 \\
22 \\
23 \\
24\end{array}$} & $N G$ \\
\hline & $\frac{N G}{1 Z G}$ \\
\hline & 128 \\
\hline
\end{tabular}

$\begin{array}{ccc}\text { TR- } 112-1 & \text { TR- } 112-2 & \text { TR-241-BX-2 } \\ 0500 & 0500 & 0500\end{array}$

\begin{tabular}{|l|l|l|}
\hline 139 & & 71 \\
\hline 39 & & 72 \\
\hline 129 & & 21 \\
\hline 191 & 91 & 67 \\
\hline 109 & & 69 \\
\hline 111 & & 56 \\
\hline 111 & & 59 \\
\hline 111 & & 53 \\
\hline 111 & & $5-3$ \\
\hline 102 & & 53 \\
\hline 122 & & 50 \\
\hline
\end{tabular}

122

123

$1 / 0$

INSTRUCTIONS:

1. F111 in a11 blanks

2. If data are not avallnble, give reason under Remarks.

3. If recording instruments, thermocouples, etc. are not runctioning properly, report this to supervision.

4. Cirele all results that are out of process ilmits or are otherwise questionable.

5. All suggestions, formal or Informal, will be given prorupt attent1on.
Record on $8-4$ Shift

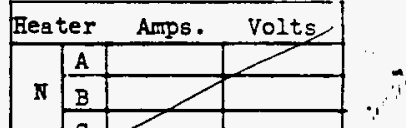

$\mathrm{s}$

w

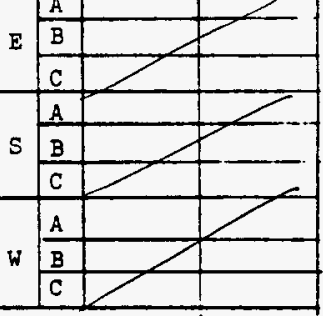




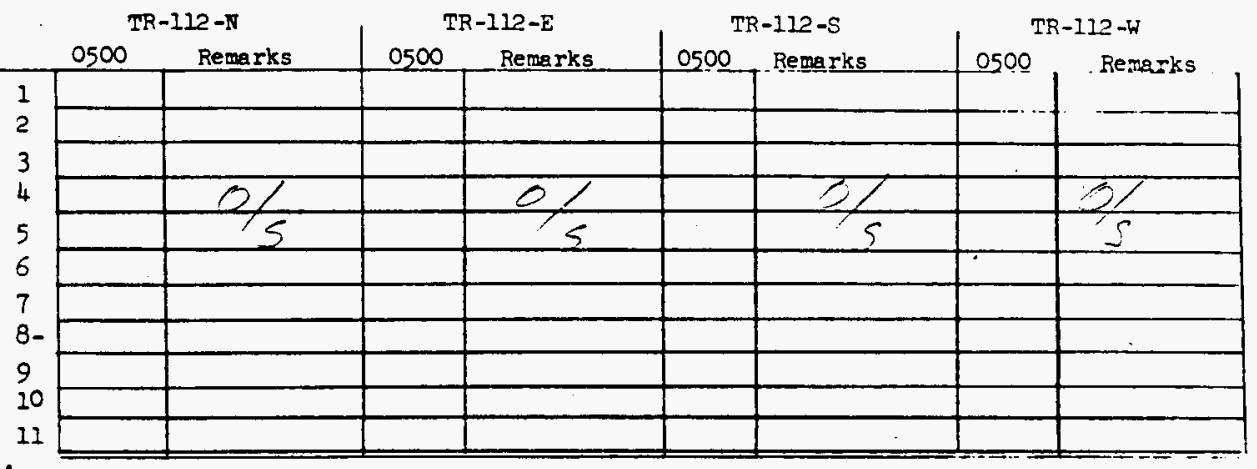

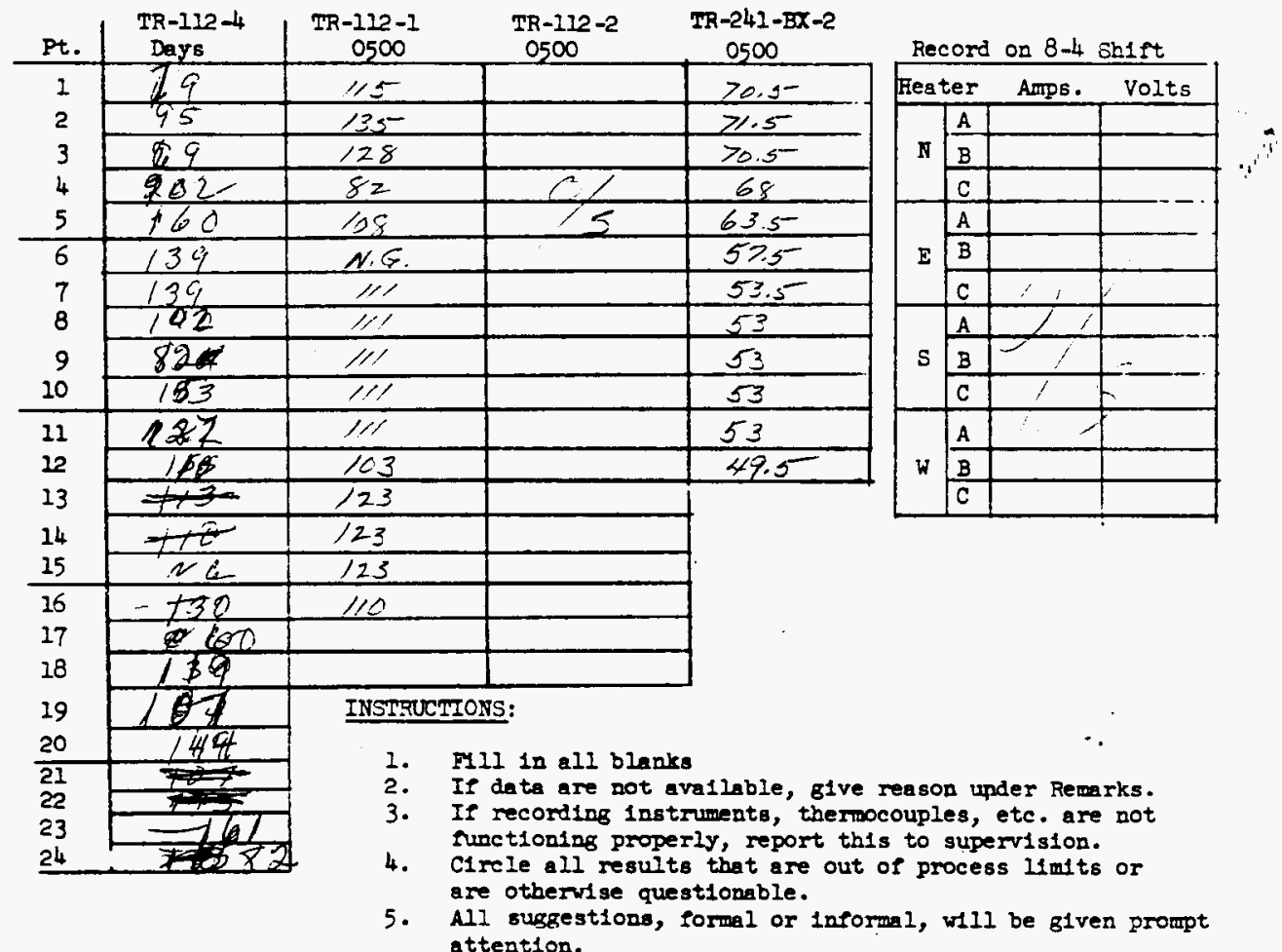


ITS-2 TEMPERATURES

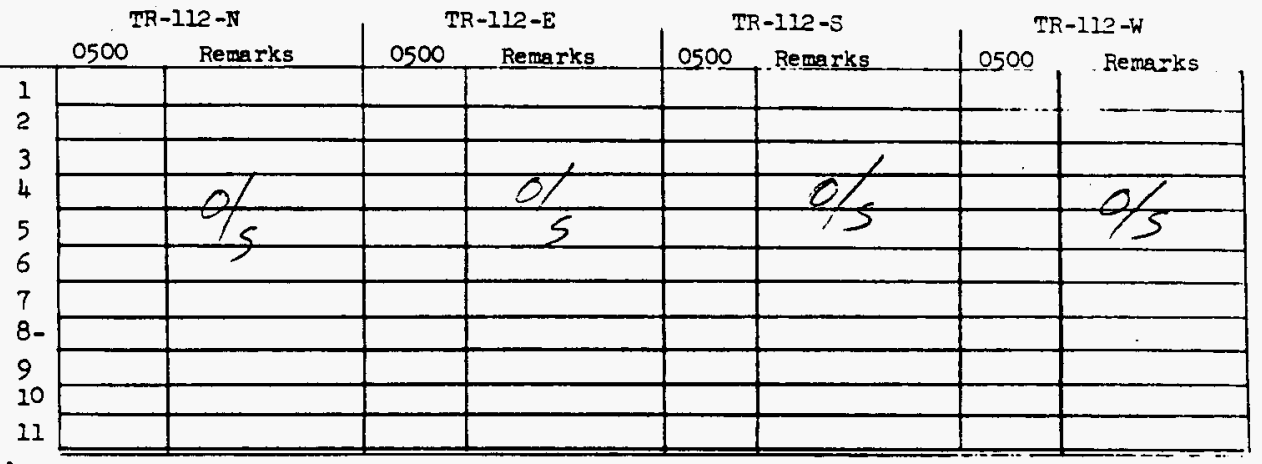

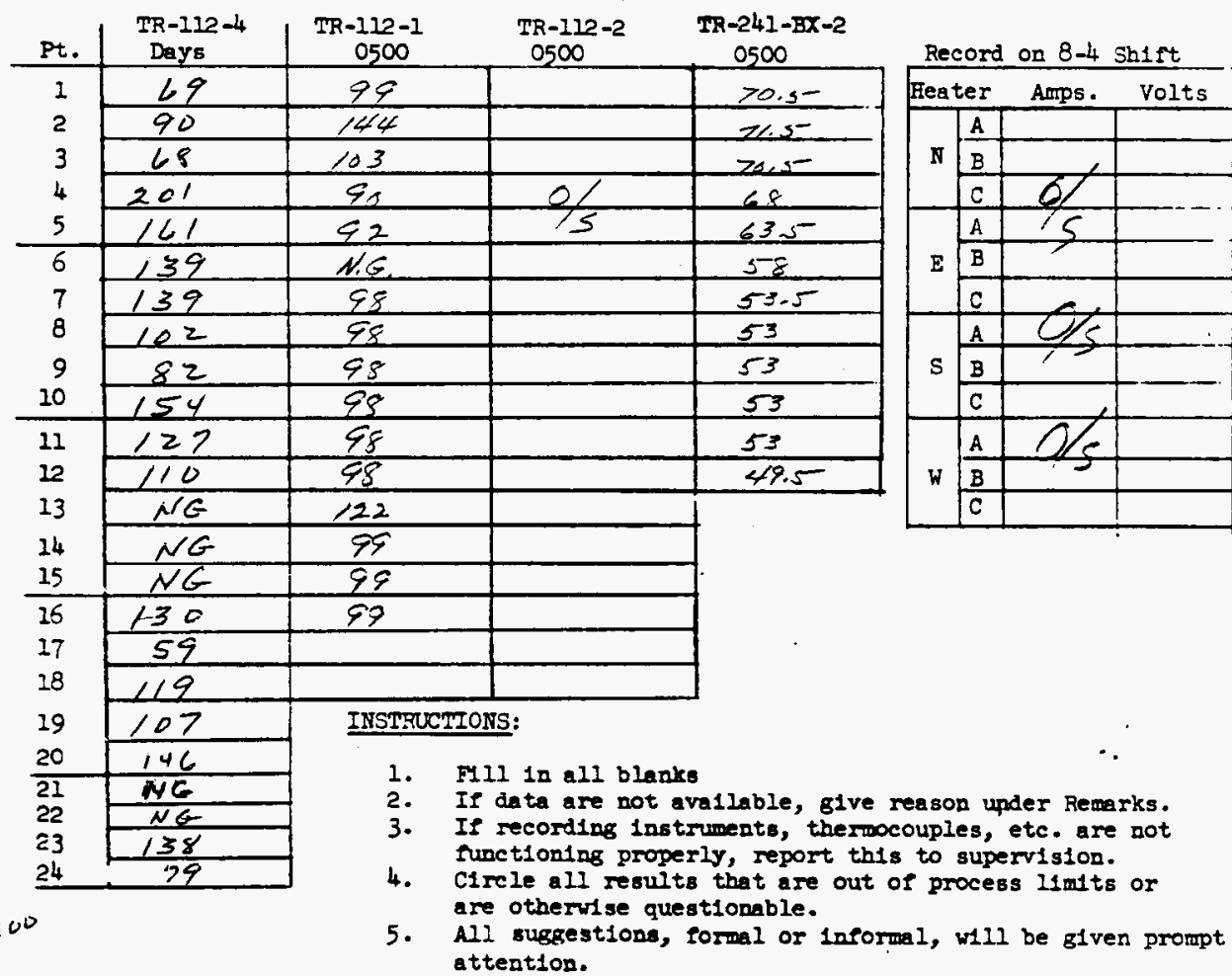




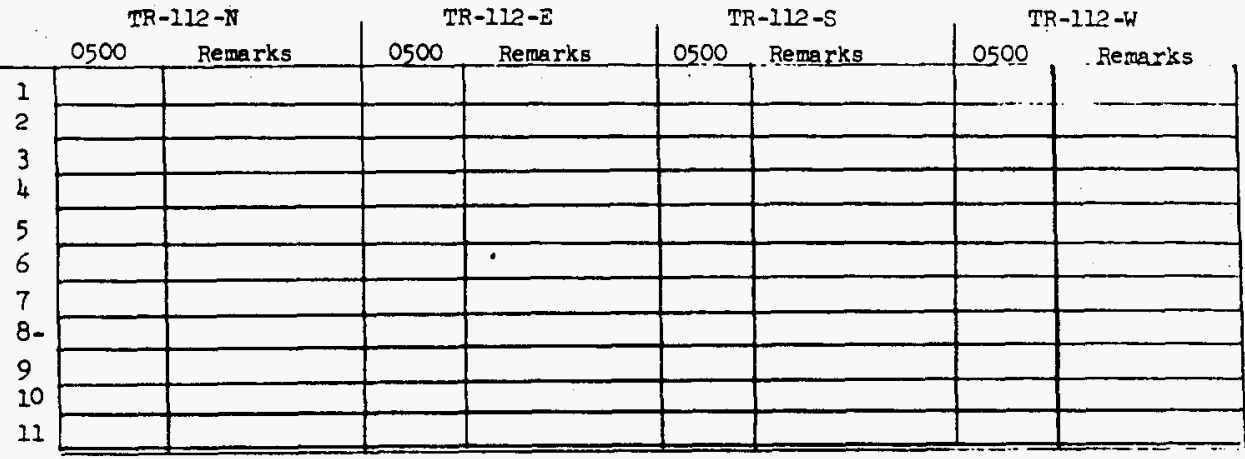

\begin{tabular}{|c|c|c|c|c|c|c|c|c|}
\hline Pt. & $\begin{array}{c}\text { TR }-112-4 \\
\text { Deys }\end{array}$ & $\begin{array}{c}\text { TR- } 112-1 \\
0500 \\
\end{array}$ & $\begin{array}{c}T R-112-2 \\
0500\end{array}$ & $\begin{array}{c}T R-24 I-B X-2 \\
0500 \\
\end{array}$ & \multicolumn{3}{|c|}{ Record on 8-4 } & Sh1ft \\
\hline 1 & 70 & 110 & & 20 & \multicolumn{2}{|c|}{ Heater } & Amps. & Volts \\
\hline 2 & 81 & $\angle 36$ & & .70 & \multirow{3}{*}{$\pi$} & A & & \\
\hline 3 & 69 & 120 & & 20 & & $B$ & & \\
\hline 4 & 201 & 90 & & 68 & & $c$ & & \\
\hline 5 & 16.1 & 105 & & 64 & \multirow{3}{*}{$\boldsymbol{E}$} & $\mathrm{A}$ & & \\
\hline 6 & 140 & n.G. & & 58 & & $B$ & & \\
\hline 7 & 140 & 110 & & 54 & & $c$ & & \\
\hline 8 & 102 & 110 & & 53 & \multirow{3}{*}{$s$} & A & & \\
\hline 9 & 83 & 109 & & 53 & & $B$ & & \\
\hline 10 & 154 & 109 & & 53 & & C & & \\
\hline 11 & 127 & 103 & & 52 & \multirow{3}{*}{ W } & A & & \\
\hline 22 & 110 & 103 & & 49 & & $\bar{B}$ & & \\
\hline 13 & $N G$ & 122 & & & & C & & 1 \\
\hline 14 & $M G$ & 115 & & & & & & \\
\hline 15 & $N G$ & 115 & & & & & & \\
\hline 16 & 130 & 165 & & & & & & \\
\hline 27 & 63 & & & . & & & & \\
\hline 18 & 119 & & & & & & & \\
\hline 19 & 107 & \multirow{2}{*}{\multicolumn{2}{|c|}{ INSTRUCTIONS: }} & & & & & . \\
\hline 20 & 144 & & & & & & & \\
\hline
\end{tabular}

1. FIIl in all blanks

2. If data are not avallable, give reason upder Remarks.

3. If recoriling 1nstruments, thernocouples, etc. are not runctioning properly, report this to supervision.

4. C1rele all results that are out of process $14 \mathrm{mits}$ or are otherwlse questionable.

5. All suggestions, formal or 10formel, w111 be given prompt attention. 


\begin{tabular}{|c|c|c|c|c|c|c|}
\hline \multicolumn{7}{|c|}{ DISTRIBUTION SHEET } \\
\hline To & \multirow{2}{*}{\multicolumn{4}{|c|}{$\begin{array}{l}\text { From } \\
\text { Remote System and Sensor } \\
\text { Applications }\end{array}$}} & \multicolumn{2}{|l|}{ Page 1 of 1} \\
\hline $\begin{array}{l}\text { Remote System and Sensor } \\
\text { Applications }\end{array}$ & & & & & \multicolumn{2}{|c|}{ Date $7 / 29 / 96$} \\
\hline \multirow{2}{*}{\multicolumn{5}{|c|}{$\begin{array}{l}\text { Project Title/Work Order } \\
\text { Sensor/Analytical Technology Projects }\end{array}$}} & \multicolumn{2}{|c|}{ EDT No. 617410} \\
\hline & & & & & ECN No. & \\
\hline Name & & MSIN & $\begin{array}{l}\text { Text } \\
\text { With All } \\
\text { Attach. }\end{array}$ & Text Only & $\begin{array}{l}\text { Attach./ } \\
\text { Appendix } \\
\text { Only }\end{array}$ & $\begin{array}{l}\text { EDT/ECN } \\
\text { Only }\end{array}$ \\
\hline $\begin{array}{l}\text { H. Babad } \\
\text { W. B. Barton } \\
\text { C. H. Brevick } \\
\text { R. J. Cash } \\
\text { M. D. Crippen } \\
\text { D. R. Dickinson } \\
\text { D. W. Jeppson } \\
\text { N. W. Kirch } \\
\text { D. J. McCain } \\
\text { J. E. Meacham } \\
\text { F. R. Reich } \\
\text { D. A. Reynolds } \\
\text { Centra? Files (original +l) }\end{array}$ & & $\begin{array}{l}\text { S7-14 } \\
\text { R2-11 } \\
\text { S3-10 } \\
\text { S7-14 } \\
\text { L5-31 } \\
\text { L5-31 } \\
\text { L5-31 } \\
\text { R2-11 } \\
\text { R2-12 } \\
\text { S7-14 } \\
\text { L5-55 } \\
\text { R2-11 } \\
\text { A3-89 }\end{array}$ & $\begin{array}{l}x \\
x \\
x \\
x \\
x \\
x \\
x \\
x \\
x \\
x \\
x \\
x \\
x\end{array}$ & & & \\
\hline
\end{tabular}

